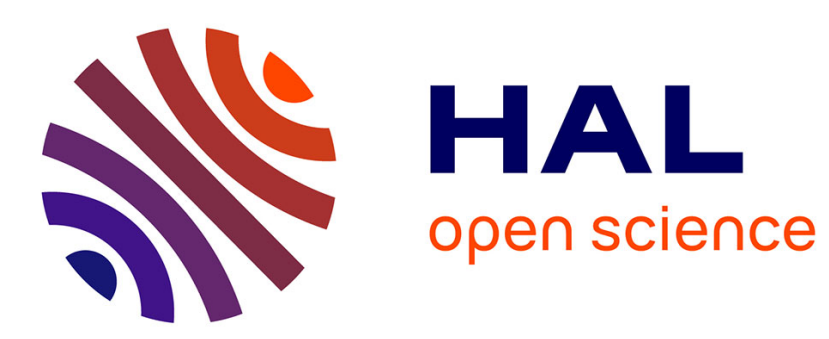

\title{
On the order induced by a set of rays. Application to the probing of non convex polygons
}

\author{
Panagiotis Alevizos, Jean-Daniel Boissonnat, Mariette Yvinec
}

\section{To cite this version:}

Panagiotis Alevizos, Jean-Daniel Boissonnat, Mariette Yvinec. On the order induced by a set of rays. Application to the probing of non convex polygons. [Research Report] RR-0927, INRIA. 1988. inria-00075629

\section{HAL Id: inria-00075629 \\ https://hal.inria.fr/inria-00075629}

Submitted on 24 May 2006

HAL is a multi-disciplinary open access archive for the deposit and dissemination of scientific research documents, whether they are published or not. The documents may come from teaching and research institutions in France or abroad, or from public or private research centers.
L'archive ouverte pluridisciplinaire HAL, est destinée au dépôt et à la diffusion de documents scientifiques de niveau recherche, publiés ou non, émanant des établissements d'enseignement et de recherche français ou étrangers, des laboratoires publics ou privés. 


\section{Rapports de Recherche}

6.1.

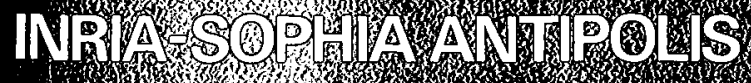

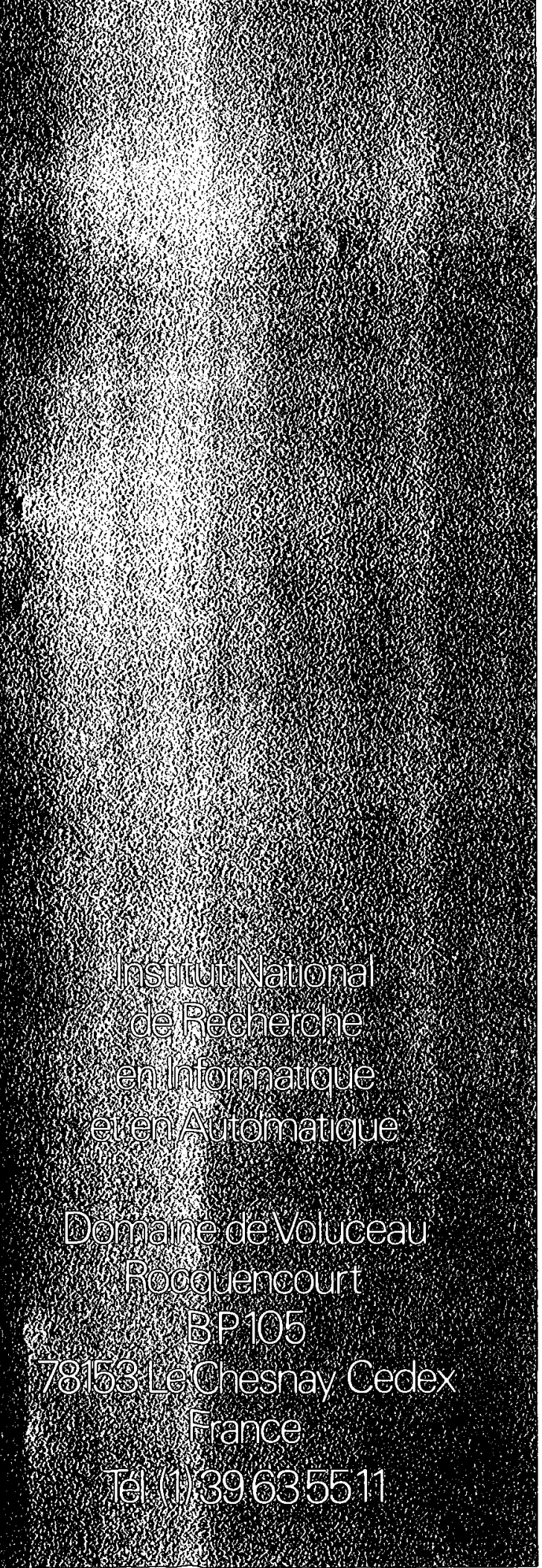

$\mathbf{N}^{\circ} 927$

Programme 6

ON THE ORDER INDUCED BY A SET OF RAYS

APPLICATION TO THE PROBING
OF NON CONVEX POLYGONS




\title{
On the order induced by a set of rays Application to the probing of non convex polygons
}

\author{
P. Alevizos* \\ Department of Mathematics \\ University of Patras \\ 26110 Patras, Greece
}

\author{
J.D. Boissonnat ${ }^{\dagger}$ \\ INRIA Sophia Antipolis \\ 2004 Route des Lucioles \\ 06565 Valbonne, France
}

M. Yvinec

Laboratoire d'Informatique

Ecole Normale Supérieure ${ }^{\ddagger}$

45 Rue d'Ulm

75230 Paris, France

\begin{abstract}
We present algorithms to reconstruct the planar cross section of a simply connected object from data points measured by rays. The rays are semi-infinite curves representing, for example, the laser beam or the articulated arms of a robot moving around the object. This paper shows that the information provided by the rays is crucial (though generally neglected) when solving 2-dimensional reconstruction problems. The main property of the rays is that they induce a total order on the measured points. This order is shown to be computable in optimal time $O(n \log n)$. The algorithm is fully dynamic and allows the insertion or the deletion of a point in $O(\log n)$ time.

From this order a polygonal approximation of the object can be deduced in a straightforward manner. However, if not enough data are available or if the points belong to several connected objects, this polygonal approximation may not be a simple polygon or may intersect the rays. This can be checked in $O(n \log n)$ time.

The order induced by the rays can also be used to find a strategy for discovering the exact shape of a simple (but not necessarily convex) polygon by means of a minimal number of probes. When each probe outcome consists of a contact point, a ray measuring that point and the normal to the object at the point, we have shown that $3 n-3$ probes are necessary and sufficient if the object has $n$ non colinear edges. Each probe can be determined in $\mathrm{O}(\log n)$ time yielding an $\mathrm{O}(n \log n)$-time $\mathrm{O}(n)$-space algorithm. When each probe outcome consists of a contact point and a ray measuring that point but not the normal, the same strategy can still be applied. Under a mild condition, $5 n-2$ probes are sufficient to discover the shape of the object in this case and, without this condition, $5 n-2$ probes allow to discover a shape which is almost surely the actual shape of the object.
\end{abstract}

\footnotetext{
*This work was done while this author was visiting INRIA Sophia-Antipolis

this author has been partly supported by the CEE ESPRIT Project P-940

'Unité de recherche associée au CNRS (URA 725)
} 


\title{
A propos de l'ordre induit par un ensemble de rayons Application au sondage de polygones non
convexes
}

\author{
P. Alevizos \\ Department of Mathematics \\ University of Patras \\ 26110 Patras, Greece
}

\author{
J.D. Boissonnat \\ INRIA Sophia Antipolis \\ 2004 Route des Lucioles \\ 06565 Valbonne, France
}

\author{
M. Yvinec \\ Laboratoire d'Informatique \\ Ecole Normale Supérieure \\ 75230 Paris, France
}

\begin{abstract}
Résumé
On présente, dans cet article, des algorithmes permettant de reconstruire une section plane d'un objet simplement connexe à partir de mesures effectuées par des rayons. Un rayon est une courbe semi-infinie représentant, par exemple, un faisceau laser ou le bras d'un robot servant à mesurer l'objet. On montre que l'information fournie par les rayons est cruciale (bien que généralement négligée) pour résoudre les problèmes de reconstruction bidimensionnels. La propriété essentielle des rayons est d'induire une relation d'ordre total sur les points mesurés. Cet ordre peut être calculé en temps $O(n \log n)$, ce qui est optimal. L'algorithme est dynamique et permet d'insérer ou de supprimer un point en temps $O(\log n)$.

On peut déduire directement de cet ordre une approximation polygonale de l'objet. Cependant, si l'on ne dispose pas de suffisamment de mesures ou si les points appartiennent à plusieurs objets, cette approximation polygonale peut ne pas être un polygone simple ou peut intersecter les rayons. Ceci peut être testé en temps $O(n \log n)$.

L'ordre induit par les rayons peut également être utilisé pour définir une stratégie capable de découvrir, à l'aide de sondages simples, la forme exacte de l'objet si celui-ci est un polygone simple (mais pas nécessairement convexe). Lorsque le résultat de chaque sondage consiste en les coordonnées du point de contact, un rayon servant à mesurer ce point et la normale à l'objet en ce point, on montre qu'il faut et qu'il suffit de sonder $3 n-3$ fois l'objet si celui-ci est constitué de $n$ arêtes non colinéaires. Chaque sondage est déterminé en temps $O(\log n)$, ce qui conduit à un algorithme de complexité $O(n \log n)$ en temps et $O(n)$ en place. Lorsque le résultat des sondages ne contient pas d'information sur la normale, la même stratégie s'applique encore. Dans ce cas et sous une hypothèse assez faible, $5 n-2$ sondages sont suffisants pour découvrir la forme; sans cette hypothèse, on montre que ces $5 n-2$ sondages permettent de découvrir une forme qui est presque surement la forme exacte de l'objet.
\end{abstract}




\section{Introduction}

Let us consider a robot equipped with a sensing device, moving around an unknown object. By means of its sensor, the robot probes the object and the problem is to reconstruct, from the probe responses, the shape or some aspect of the unknown object. A variety of subproblems can be distinguished, depending on the model of the sensor and on the constraints on the type of the object. In this paper, we restrict our attention to 2-dimensional variants of the problem and assume that the sensor probes in a plane.

Let us consider the typical situation where each probe response consists of the coordinates of a point on the boundary of the object (a "finger probe", according to Skiena's taxonomy [12]). In order to reconstruct the shape of the object, it is crucial to recover, from the probe responses, the order of the measured points as they appear on the boundary of the object. Clearly, without this order, it is impossible to infer the shape or even a reasonable approximation of the shape and we must content ourselves with heuristics or with methods that are only guaranteed to work for sufficiently dense sets of points $[4,10]$. On the other hand, having this order will allow to compute a polygonal approximation of the object by connecting the measured points in their order.

The order the points appear on the boundary of the object is, for some classes of objects, implicitly contained in the data. If the object is convex, the order is simply the order the points appear on their convex hull. The order is also implicitly contained in the data points if, as shown by O'Rourke [9], the object is an orthogonal polygon $^{1}$ (i.e., each vertex is incident to exactly one horizontal edge and one vertical edge).

In order to study more general objects, we prefer not to impose constraints on the shape of the object but, instead, to use more powerful probes. This is not to say that new sensing devices are necessarily required. In fact, this paper is motivated by the observation that, in most situations, the information necessary to recover the order is implicitly contained in the probe. More precisely, the information is contained in some rays which served measuring the points. A ray is any semi-infinite curve which has the measured point as its origin and which does not intersect the interior of the object (see Figure 1). In case of an optical device, the rays are half straight lines between the sensor (supposed to be at infinity) and the points - the optical rays. If the object is not transparent, these rays cannot intersect the interior of the object. In other situations, the robot may simply touch the object with the tip of its $\operatorname{arm}(\mathrm{s})$. In that case, the rays consist in a set of polygonal lines issued from the contact points, which represent the different positions of the $\operatorname{arm}(\mathrm{s})$ when touching the object. Notice that, in this case, the number of line segments of a polygonal line is bounded by the maximal number of articulated bodies of the robot

\footnotetext{
${ }^{1}$ In fact, O'Rourke proved the result for a family of disjoint orthogonal polygons.
} 
$\operatorname{arm}(\mathrm{s})$. A similar situation occurs when a mobile robot moves along some path until it encounters the boundary of an object. The path followed by the robot is a ray.

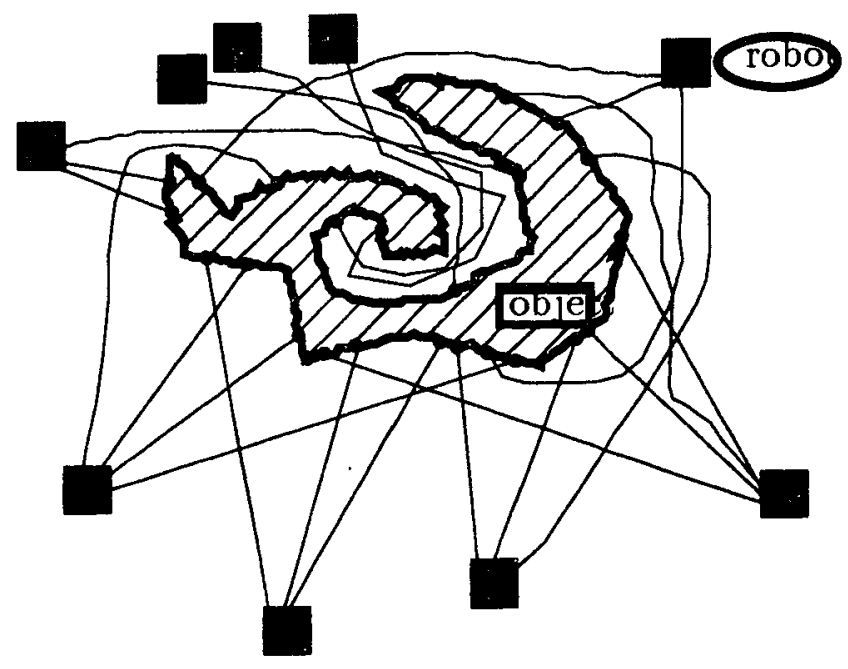

Figure 1: A robot probing an object with rays

The information provided by the rays is crucial for reconstructing shapes. Indeed, we will show in Section 2.2 that the rays induce a total order on the points, the same as the order the points appear along the boundary of the object. Moreover, this order can be computed in optimal $O(n \log n)$ time if the number of measures is $n$. The algorithm presented in Section 2.3 is fully dynamic and allows the insertion or even the deletion of a measured point in $O(\log n)$ time. From this order a polygonal approximation of the object can be deduced in a straightforward manner as mentionned above. However, if not enough data are available or if the points belong to several connected objects, this polygonal approximation may not be a simple polygon or may intersect the rays. This can be checked in $O(n \log n)$ time as is shown in Section 2.4.

When the object is known to be piece-wise linear, the order induced by the rays can also be used to find a good strategy for discovering the exact shape of the object. This problem is often referred to as the probing problem in the literature and has been studied in the case of a convex polygonal object: Cole and Yap [6] showed that the shape of a convex polygon with $n$ edges can be determined with no more than $3 n$ probes; Bernstein [3] has improved this result if the polygon is restricted to a finite set and Dobkin, Edelsbrunner and Yap [7] have considered the case of convex polytopes in multidimensionnal space. A work of synthesis of the field of geometric probing as well as a collection of new results can be found in Skiena' Ph.D. Thesis [12]. In Section 3, we show how the order induced by the rays can 
be used to generalize the results of Cole and Yap to non convex polygons with no colinear edges. It is proved that, if each probe outcome consists of a contact point, a ray measuring that point and the normal to the object at the point, $3 n-3$ probes are necessary (Section 3.4) and sufficient (Section 3.2). In Section 3.3, we prove that each probe can be determined in $\mathrm{O}(\log n)$ time yielding an $\mathrm{O}(n \log n)$-time $\mathrm{O}(n)$ space algorithm. If the normals are not available, it is proved that the same strategy can still be applied. Under a mild condition, $5 n-2$ probes are sufficient to discover the shape of the object in this case. Without this condition, $5 n-2$ probes allow to discover a shape which is almost surely the actual shape of the object.

Let us give a few preliminary notations, remarks and general assumptions which will be made through the whole paper. In the two dimensional plane, let $P$ be a set of $n$ points $p_{1} \ldots p_{n}$ and $L$ be a set of $n$ semi-infinite curves, called rays, $l_{1} \ldots l_{n}$, such that $l_{i}$ originates at point $p_{i}$. According to physical constraints, we always consider that the points of $P$ lie on the boundary of a real solid object and that the rays never intersect the interior of the object ${ }^{2}$. We assume that the $n$ points belong to a unique simply connected object without holes. From a theoretical point of view, the ray can be any simple semi-infinite continuous curve originating at a point of $P$ and ending at a point at infinity. The restriction to simple curves is in fact not essential and done for simplicity. For the purpose of practical reconstruction algorithms, we shall restrict our presentation to polygonal rays although the method works for more general curves; the last edges of these polygonal lines are supposed to be semi-infinite straight lines. Moreover, our complexity results assume that the number of segments of each ray is bounded by a constant.

\section{The contour reconstruction problem}

\subsection{Statement of the problem}

For the given set of points $P$ and the set of rays $L$, the aim is to find a polygonal approximation of the object boundary, called a polygonal contour in the sequel, which is a simple polygon having the points of $P$ as vertices and intersecting none of the rays of the set $L$. Such a polygonal contour does not exist for any given set of data $(P, L)$. There are two typical situations in which no polygonal solution to the contour problem can be found.

The first one is shown in Figure 2. In that case, there are two rays, say ray $l_{a}$ measuring point $a$ and ray $l_{b}$ measuring point $b$, which intersect in at least one point at finite distance. Such a pair of rays partitions the plane into more than one connected regions. Let $W_{a b}$ be the union of the regions which do not contain points $a$ and $b ; W_{a b}$ is the empty region if rays $l_{a}$ and $l_{b}$ do not intersect in a finite point and a simple wedge if $l_{a}$ and $l_{b}$ are straight intersecting rays. If some points, like

\footnotetext{
${ }^{2}$ For short, we will omit "the interior of" in the sequel.
} 
point $c$ in Figure 2, are measured in the region $W_{a b}$, then obviously the data points belong to more than one object because no continuous curve can join points in $W_{a b}$ to points $a$ and $b$ without intersecting ray $l_{a}$ or ray $l_{b}$. We shall say that the region $W_{a b}$ is hidden by rays $l_{a}$ and $l_{b}$.

In the sequel, we call legal a set of data $(P, L)$ for which no point lies in a hidden region. In other words, a set of data $(P, L)$ is legal if any pair of measured points $(a, b)$ can be joined by a continuous curve without intersection with the set of rays $L$ (except at points $a$ and $b$ with respectively the rays $l_{a}$ and $l_{b}$ measuring these points).

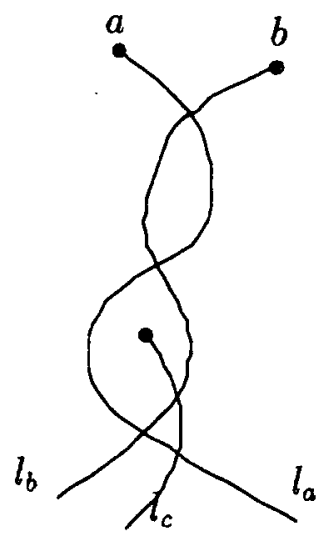

Figure 2: The case where the data points belong to more than one object

In the second situation, shown in Figure 3, the data are legal and there is a simple contour passing through all the points of $P$ and intersecting no ray of $L$ but this contour cannot be drawn with straight line segments joining the points of $P$. Such a situation arises when too few data are available: for example, in Figure 4, the addition of a new point $b$ to the set of data restore the existence of a solution to the contour problem. In that case, we say that the contour problem admits only a topological solution. A topological contour on a set of points $P$ is a cyclic ordering of the points of $P$ such that there is a simple closed curve passing through all the points of $P$ in that order and not intersecting the rays. Such a curve is called a representation of the topological contour and is oriented counterclockwise. A topological contour becomes a linear ordering of the points as soon as one particular point has been chosen as the origin on the contour. A topological contour on a set of points $P$ is a solution of the problem contour $(P, L)$ if there is a representation of this contour which has no intersection with the set of rays $L$.

In this section, we shall first prove that any legal contour problem admits a unique topological solution. Then, we present an algorithm which finds the topological solution of a legal contour problem of size $n$ in time $\mathrm{O}(n \log n)$ which is shown to be 


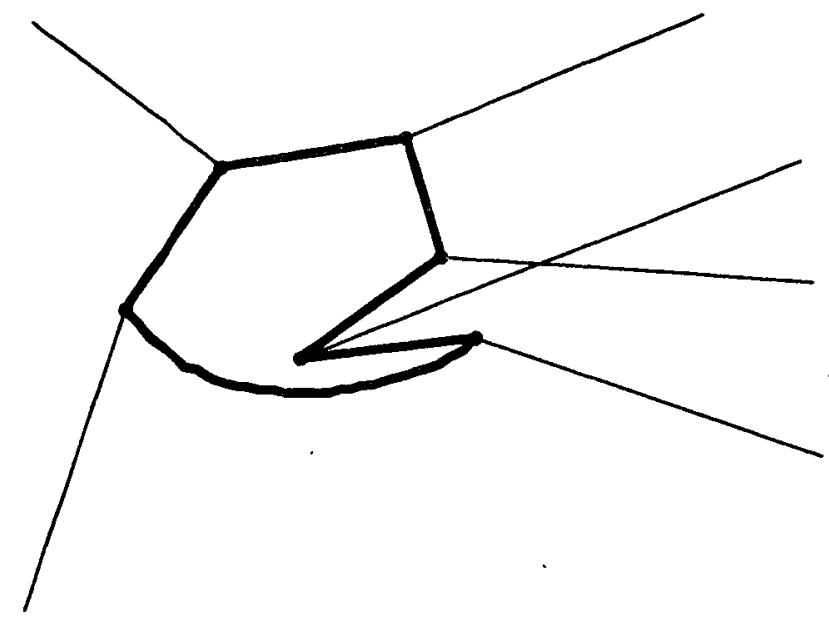

Figure 3: Too few data are available

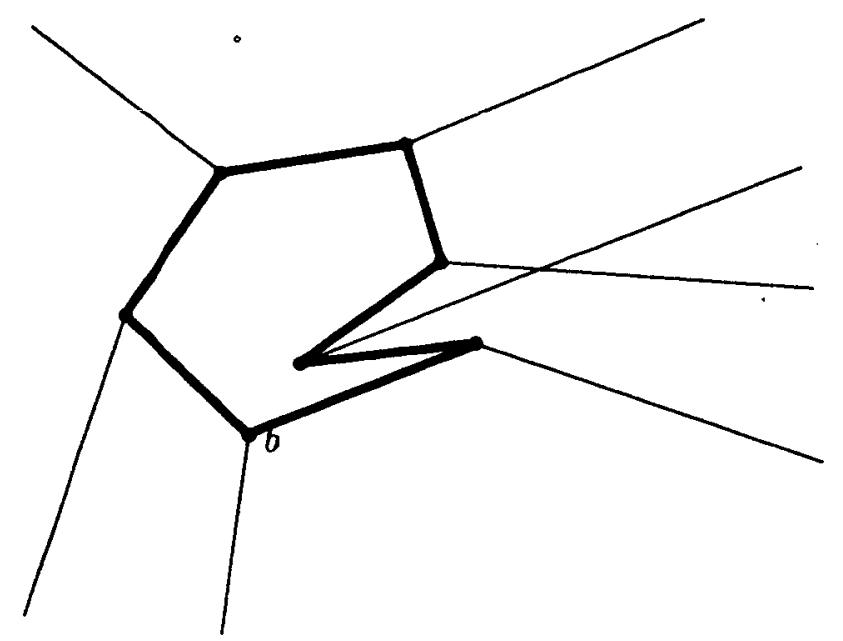

Figure 4: Adding a new point to restore the existence of a solution to the contour problem 
optimal. Furthermore, we say that a legal set of data $(P, L)$ is complete if the closed polygon which is the (unique) piece-wise linear representation of the topological solution is actually a solution of the contour problem. In case of illegal or incomplete data sets, this algorithm yields a polygon which either is not simple or intersects some of the rays. Both situations can be detected a posteriori through a serie of simple tests performed on the obtained polygon in time $O(n \log n)$. Unfortunately, in case of failure, these simple tests do not distinguish between illegal and incomplete data.

\subsection{Existence and uniqueness of the topological contour of any legal set of data}

Let $(P, L)$ be a legal set of data. We first prove a lemma which is a necessary and sufficient condition for two points $a$ and $b$ to be consecutive along the topological contour solution of the problem $(P, L)$. A few definitions are needed. For any pair of points $(a, b)$, let $\sum_{a b}$ be a simple curve joining $a$ to $b$ without intersecting the rays of $L$. The curve $\sum_{a b}$, together with the rays $l_{a}$ and $l_{b}$ measuring respectively the points $a$ and $b$ partitions the plane into three regions : the first one is the (eventually empty) region $W_{a b}$ which is hidden by rays $l_{a}$ and $l_{b}$, the other two, called $H_{a b}$ and $\vec{H}_{a b}$, arise when splitting the complementary region of $W_{a b} ; H_{a b}$ (resp., $\bar{H}_{a b}$ ) is the region to the right (resp. to the left) of $\sum_{a b}$ assumed to be oriented from $a$ to $b$ (See Figure 5).

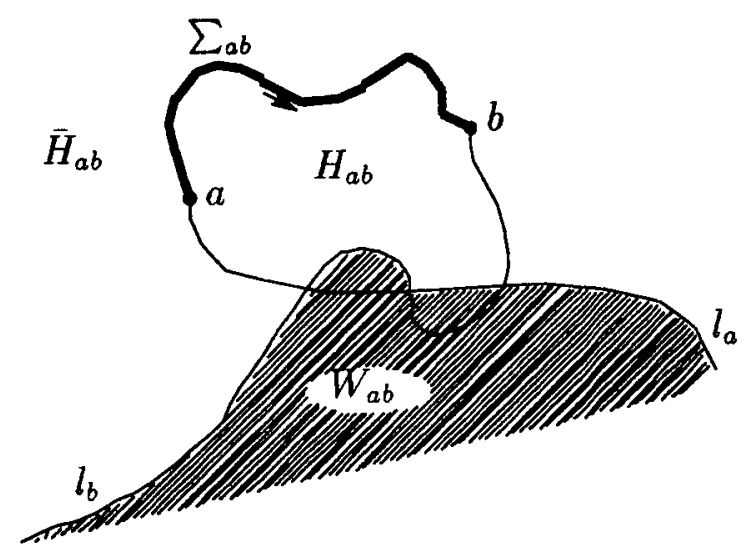

Figure 5: For the definition of $W_{a b}, H_{a b}$ and $\Sigma_{a b}$

Lemma 1 The two points $a$ and $b$ are consecutive on the topological contour solution of the problem $(P, L)$ if and only if there exists a simple curve $\sum_{a b}$ such that :

1) the curve $\sum_{a b}$ intersects no ray of $L$; 


\section{2) the region $H_{a b}$ contains no point of $P$ in its interior.}

Proof : Assume first that $a$ and $b$ are consecutive points on the topological contour solution of the problem $(P, L)$. Let $\sum$ be a representation of this topological solution and let $\sum_{a b}$ be the part of $\sum$ joining $a$ to $b$. Then, by definition, $\sum_{a b}$ intersects no ray of $L$ and furthermore the interior of the region $H_{a b}$ is totally included in the outside of the object bounded by $\sum$ and thus contains no point of $P$.

Conversely, assume that there is a curve $\sum_{a b}$ joining $a$ to $b$ and satisfying both conditions of Lemma 1. Assume for a contradiction that there is a solution of the problem $(P, L)$ in which $a$ and $b$ are not consecutive. Let $\Sigma^{\prime}$ be a representation of this topological solution; then $\Sigma^{\prime}$ goes at least through another point $c$ of $P$ between $a$ and $b$; let $\sum_{a c b}^{\prime}$ be the part of $\sum^{\prime}$ joining $a$ to $b$ (See Figure 6). The point $c$, which cannot belong to the hidden region $W_{a b}$, either belongs to $H_{a b}$, which contradicts the second condition of Lemma 1 , or belongs to $\bar{H}_{a b}$. In this latter case, the ray $l_{c}$, measuring $c$, which is on the right side when going from $a$ to $b$ on $\sum_{a c b}^{\prime}$, must necessarily intersect $\sum_{a b}$ which contradicts the first condition of Lemma 1 .

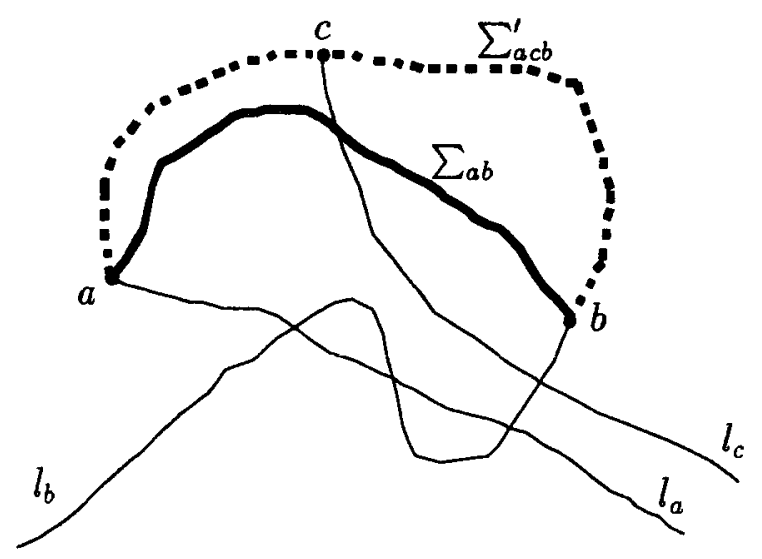

Figure 6: For the proof of Lemma 1

Lemma 1 can be generalized in a straightforward way. Let $\sum_{q_{1} \ldots q_{m}}$ be a simple curve joining the points $q_{1}, \ldots, q_{m}$ of $P$ in that order. A necessary and sufficient condition for $\sum_{q_{1} \ldots q_{m}}$ to be part of a representation of the topological solution of the problem $(P, L)$ is that:

1. $\sum_{q_{1} \ldots q_{m}}$ intersects no ray of $L$ (except the ray $l_{i}$ measuring point $q_{i}$ at this point for $i=1, \ldots, m)$

2. $H_{q_{1} \ldots q_{m}}=\bigcup_{i=1}^{m-1} H_{q_{i} q_{i+1}}$ contains no point of $P$. 
Let us come now to the existence and uniqueness of a topological solution for any legal contour problem. More precisely, we shall prove the following theorem :

Theorem 1 For any legal set of data $(P, L)$, the set of rays $L$ induces a total cyclic ondering of the points of $P$. This total cyclic order is the unique topological solution of the contour problem $(P, L)$.

Proof : Let us choose the point $a$ of $P$ as the origin. For any point $q_{i}$ of $P-\{a\}$, let $\sum_{a q_{i}}$ be a simple curve joining $a$ to $q_{i}$ without intersecting the rays of $L$. For any pair $\left(q_{i}, q_{j}\right)$ of points of $P-\{a\}$, either $q_{i}$ lies inside the region $H_{a q_{j}}$ or $q_{j}$ lies inside the region $H_{a q_{i}}$. Indeed, assume that $q_{j}$ does not lie inside the region $H_{a q_{i}}$, then $q_{j}$ lies in $\bar{H}_{a q_{i}}$ because the set of data is legal. Now the ray $l_{j}$ of $q_{j}$ does not intersect $\sum_{a q_{i}}$ which implies that $q_{i}$ lies in the region $H_{a q_{j}}$ (see Figure 7 ). We shall denote by $q_{i} \prec{ }_{a} q_{j}$ the relation " $q_{i}$ lies in $H_{a q_{j}}$ ". Because the data are legal, no point of $P$ lie in the hidden region $W_{q_{i} q_{j}}$. Furthermore, if the two curves $\sum_{a q_{i}}$ and $\sum_{a q_{j}}$ intersect each other in some points distinct from $a$, no point of $P$ can lie in the interior of a region totally bounded by these two curves because the ray of such a point would necessarily intersect $\sum_{a q_{i}}$ or $\sum_{a q_{j}}$. Thus, the relation $q_{i} \prec_{a} q_{j}$ is equivalent to the fact that the subset $P \bigcap H_{a q_{i}}$ is included in the subset $P \cap H_{a q}$, which proves the transitivity of this relation. The relation $\prec_{a}$ is therefore a total order relation on the points of $P-\{a\}$.

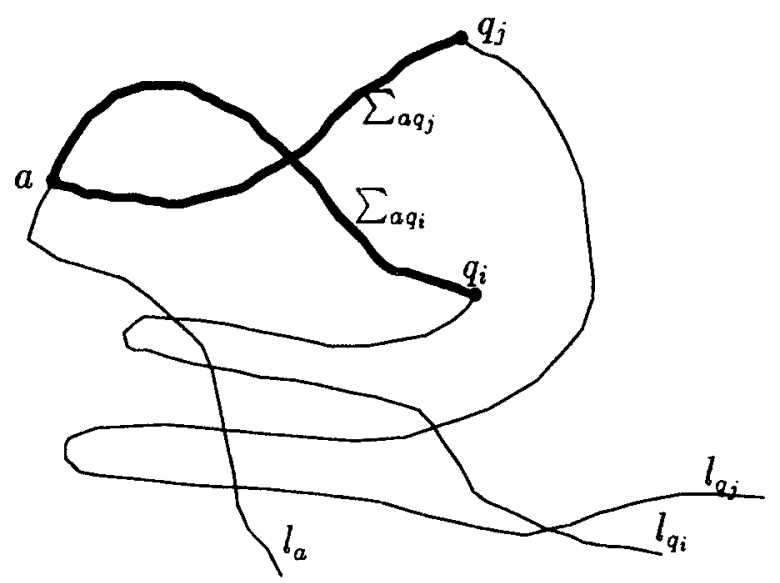

Figure 7: $q_{i}$ lies in the region $H_{a q_{j}}$

The order relation $\prec_{a}$ is independent of the actual choice of the curves $\sum_{a q_{i}}$ and $\sum_{q_{i} q_{j}}$ provided that they do not intersect the rays of $L$. Indeed any choice of these curves yields the same subsets of points $P \cap H_{a q_{i}}$ and $P \cap H_{q_{i} q_{j}}$ because no point of $P$ lie in the interior of a region of the plane totally bounded by these curves. Let 
us now show that this ordering is a topological solution of the contour problem and that this solution is unique. Let $q_{1}$ be the minimum of $P-\{a\}$ for the order $\prec_{a}$; then obviously $H_{a q_{1}}$ contains no point of $P$ and $q_{1}$ is the unique point of $P$ having this property. Then, from Lemma 1 , we know that the successor of $a$ on the contour is $q_{1}$ and that it is uniquely defined. Now for any pair $\left(q_{i}, q_{j}\right)$ of points of $P-\{a\}$ such that $q_{i} \prec_{a} q_{j}$ we have (see Figure 8):

$$
P \cap H_{a q_{j}}=\left(P \cap H_{a q_{i}}\right) \bigcup\left\{q_{i}\right\} \bigcup\left(P \cap H_{q_{i} q_{j}}\right)
$$

where the unions are disjoint unions. This is easily shown using the same kind of arguments as above. Equivalently, if $\operatorname{card}()$ is the number of elements of a set, we have :

$$
\operatorname{card}\left(P \cap H_{a q_{j}}\right)=\operatorname{card}\left(P \cap H_{a q_{i}}\right)+1+\operatorname{card}\left(P \cap H_{q_{i} q_{j}}\right)
$$

Then the successor of $q_{i}$ in the order $\prec_{a}$ is the unique point $q_{j}$ such that

$$
\operatorname{card}\left(P \cap H_{a q_{j}}\right)=\operatorname{card}\left(P \bigcap H_{a q_{i}}\right)+1
$$

which is equivalent to say that $\operatorname{card}\left(P \cap H_{q_{i} q_{j}}\right)=0$. Thus, from Lemma 1 , the successor of $q_{i}$ on the topological solution of the problem $(P, L)$ is uniquely defined as being the successor of $q_{i}$ in the order $\prec_{a}$.

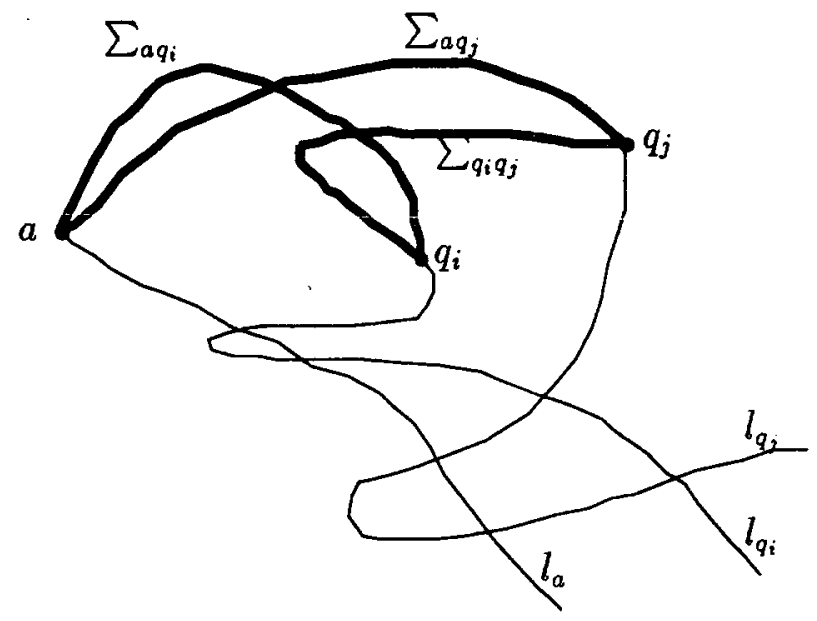

Figure 8: The order relation is independent of the actual choice of $\sum_{a q_{i}}$ and $\sum_{q_{i} q_{j}}$

\subsection{An $\mathbf{O}(n \log n)$ algorithm for the contour reconstruction problem}

In this section we propose an algorithm which, for any legal set of data $(P, L)$ provides the ordering of the points of $P$ corresponding to the topological solution 
of the contour problem $(P, L)$. This algorithm, called Algorithm Contour, is in fact an incremental sorting algorithm, where the data points are introduced one by one into a balanced tree structure (namely, an AVL tree), which maintains the points processed so far, in the order induced by their measuring rays. The data structure uses $\mathrm{O}(n)$ space and the whole algorithm runs in time $O(n \log n)$ which is shown to be optimal. Moreover, the data structure is fully dynamic and allows the insertion as well as the deletion of data points in time $O(\log n)$ per insertion or deletion. Our analysis assumes that the rays are polygonal lines with at most $k$ edges, the last edge of each ray being a semi-infinite straight line. In fact, the same algorithm and its complexity analysis can be applied to more general ray curves as long as two rays intersect in at most a bounded number of points each of which can be found in constant time.

The correctness of this incremental algorithm relies on the fact, stated in Lemma 2 just below, that the topological solution of the contour problem for any subset $\left(P^{\prime}, L^{\prime}\right)$ of the legal set of data $(P, L)$ is a subsequence of the topological solution of the problem $(P, L)$.

Lemma 2 Let $\left(P^{\prime}, L^{\prime}\right)$ be any subset of the legal set of data $(P, L)$. The topological solution of the contour problem $\left(P^{\prime}, L^{\prime}\right)$ is a subsequence of the solution of the problem $(P, L)$.

Proof : Let $a$ be a point of $P$ chosen as the origin. Let $q_{i}$ and $q_{j}$ be two points of $P^{\prime}$ such that $q_{i}$ precedes $q_{j}$ in the order induced on the set of points $P$ by the set of rays $L\left(q_{i} \prec_{a} q_{j}\right)$. Then for any choice of the curves $\sum_{a q_{i}}$ and $\sum_{a q_{j}}$ which do not intersect the rays of the set $L$, we have :

$$
P \bigcap H_{a q_{i}} \subset P \bigcap H_{a q_{j}}
$$

which implies that

$$
P^{\prime} \bigcap H_{a q_{i}} \subset P^{\prime} \bigcap H_{a q_{j}}
$$

This, from the proof of Theorem 1 , is equivalent to saying that $q_{i}$ precedes $q_{j}$ in the order induced by the subset of rays $L^{\prime}$ on the subset of points $P^{\prime}$.

Let us now present one of the basic ingredients of our method. It is an elementary algorithm, called Function Threepoints, which solves the contour problem for a reduced data set consisting of three measured points.

Let $a, b, c$ be three points measured, respectively, by the rays $l_{a}, l_{b}$ and $l_{c}$ which together form a legal data set. Function Threepoints will answer the following question: which one of the two sequences $a b c$ or $a c b$ is the solution of the contour problem $\left(\{a, b, c\},\left\{l_{a}, l_{b}, l_{c}\right\}\right)$. To answer this question, Function Threepoints searches which one of the two points $b$ or $c$ is the successor of point $a$ on the contour by constructing 
a simple curve $\sum$ which joins point $a$ to either $b$ or $c$ and satisfies the condition of Lemma 1.

The curve $\sum$ is constructed as follows : let us consider the generalized arrangement formed by the three rays $l_{a}, l_{b}$ and $l_{c}$ together with the circle $\Gamma$ which is the line at infinity. This arrangement is made of generalized edges which are the connected portions delimited on each of these four curves by its intersections with the three others. The curve $\sum$ follows some of the edges of this arrangement always staying at a small distance $\epsilon$ of the rays and turning left each time an intersection point is encountered. More precisely, the curve $\sum$, starting from point $a$, follows ray $l_{a}$ towards infinity at distance $\epsilon$ on the left side of $l_{a}$ (assumed to be oriented from $a$ to infinity) until it reaches (at distance $\epsilon$ ) the first intersection point on $l_{a}$. At this point, $\sum$ turns left and then follows the intersecting curve at distance $\epsilon$ until the next intersection point is reached and so on. (See Figure 9 for examples).

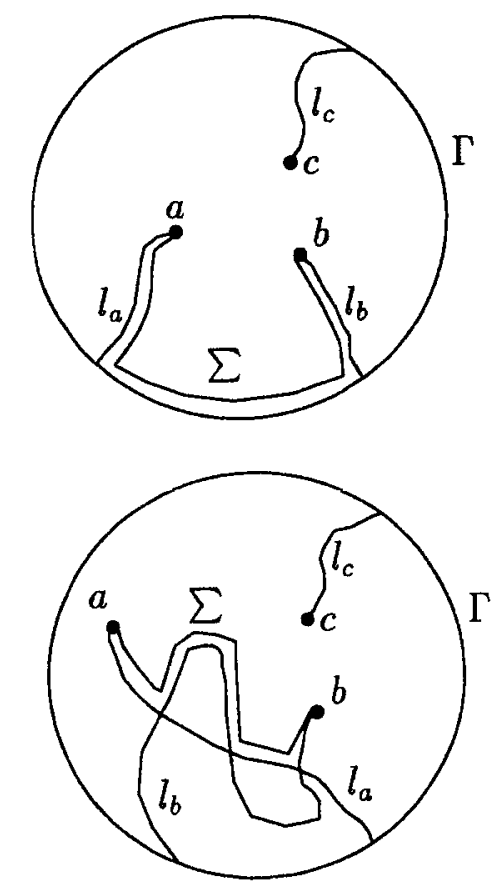

Figure 9: Illustrations of the construction of $\Sigma$ by Function Threepoints

By construction, the curve $\sum$ is a simple curve which follows each edge of the arrangement at most once and cannot return to the point $a$ which is at the origin of a ray. As the number of edges of each ray is bounded by a constant, the same holds for the number of edges in the arrangement. Thus $\sum$ will necessarily reach either point $b$ or point $c$ after a finite bounded number of left turns. 
The curve $\sum$ intersects none of the ray $l_{a}, l_{b}$ and $l_{c}$ and delimit with the rays measuring its endpoints a region $H$ which is such that $H-\left(W_{a b} \bigcup W_{b c} \bigcup W_{c a}\right)$ is arbitrarily small and thus includes no point of the three points set. Thus, it follows from Lemma 1, that the point reached by $\sum$ is the successor of point $a$ on the contour.

From the complexity point of view, since the number of edges of each ray is bounded, the number of intersections between two rays is also bounded and the above arrangement, together with the path $\sum$, can be constructed in constant time. This discussion can be summarized by the following pseudo-code for function Threepoints and the subsequent Lemma :

\section{Function Threepoints $\left(\{a, b, c\},\left\{l_{a}, l_{b}, l_{c}\right\}\right)$}

input : three points $a, b, c$ and their measuring rays $l_{\mathfrak{a}}, l_{b}, l_{c}$ forming together a legal data set

output : The solution of this three point contour problem

1. Construct the arrangement formed by the three rays $l_{a}, l_{b}, l_{c}$ together with the line at infinity $\Gamma$;

2. Construct the path $\sum$ joining $a$ to either $b$ or $c$ as described above;

3. If $\sum$ reaches point $b$ the solution is $a b c$ else the solution is $a c b$.

Lemma 3 Function Threepoints provides the topological solution of a three points contour problem in constant time if the number of intersections between two rays is bounded and if each one of these intersections can be found in constant time.

Let us come now to the description of Algorithm Contour. In this algorithm, the function Threepoints plays the role of the comparison function which is needed by any sorting algorithm. Indeed, in view of Lemma 3, a call to this function for any three points subset $\left(\{a, b, c\},\left\{l_{a}, l_{b}, l_{c}\right\}\right)$ of $(P, L)$ provides the ordering of the three points $a, b$ and $c$ on the contour solution of the problem $(P, L)$; in other words, this function compares $b$ to $c$ according to the total order induced by the set rays $L$ once point $a$ has been chosen as its origin.

Before going into the details of the algorithm, we have to make precise the data structure used to maintain the topological contour which is the current solution of the subproblem corresponding to the already processed points. This structure is a balanced tree, namely an AVL tree, which we shall call the contour tree. In each node $V$ of this tree we store a pair of two processed points $(b(V), e(V))$ such that (see Figure 10) : 
1. The leaves of the contour tree store the (topological) edges of the current contour. That is, if $V$ is a leaf, the two points $b(V)$ and $e(V)$ are consecutive in that order along the current contour. Furthermore, the order of the leaves in a traversal of the tree is the order of the edges on the contour.

2. For any internal node $V$, except for the root of the tree, the points $b(V)$ and $e(V)$ are respectively the first and last point of the portion of the contour spanned by the leaves of the subtree rooted at node $V$. For consistency, the point $a$ which is chosen as origin on the contour is stored twice in the root of the contour tree, that is : $b($ root $)=e($ root $)=a$.
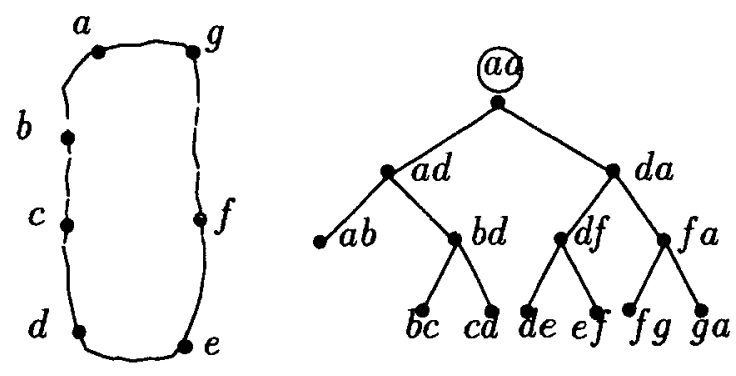

Figure 10: A contour tree

Here is the description of Algorithm Contour.

Step 1: In an initial step, three points $a, b, c$ are chosen from the set $P$, one of which, say point $a$ for instance, is to be the origin for the description of the contour. A first call to the function Threepoints is issued with arguments corresponding to these three points and the contour tree is initiated with three leaves corresponding to the edges $a b, b c$ and $c a$, or $a c, c b$ and $b a$ in accordance with the permutation of $a, b$ and $c$ output by this function. For each of the two possible permutations, two initial configurations of the contour tree are possible but that is of no importance; these configurations are shown in Figure 11 in the case that the ordering of the three initial points is $a b c$.

Step 2: The other points of the data set are introduced one by one in the structure. To introduce point $x$ measured by ray $l_{x}$, we follow a path from the root of the contour tree to the leaf storing the edge of the current contour where point $x$ is to be inserted. At each traversed internal node, the path is guided further by a call to function Threepoints. The reached leaf, $V$, is turned into an internal node with a leaf corresponding to the edge $(b(V), x)$ as leftson and a leaf corresponding to $(x, e(V))$ as rightson. 

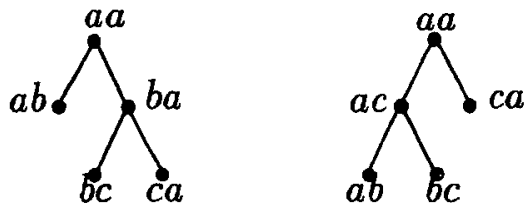

Figure 11: Initial configurations of a contour tree

After each such insertion, the tree is rebalanced using the rotation process common to all AVL trees. In addition, during an elementary right or left rotation, we have to update the points $e(V)$ and $b(V)$ of one of the concerned nodes. This is done by using for instance the label of its sons as it is illustrated in Figure 12 which shows the two possible cases of elementary rebalancing processes.

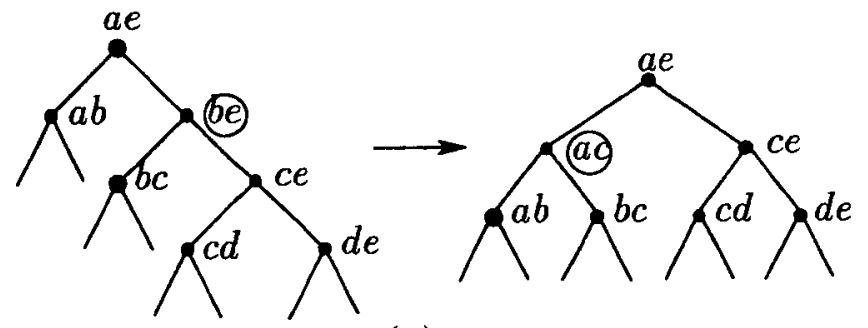

(a)

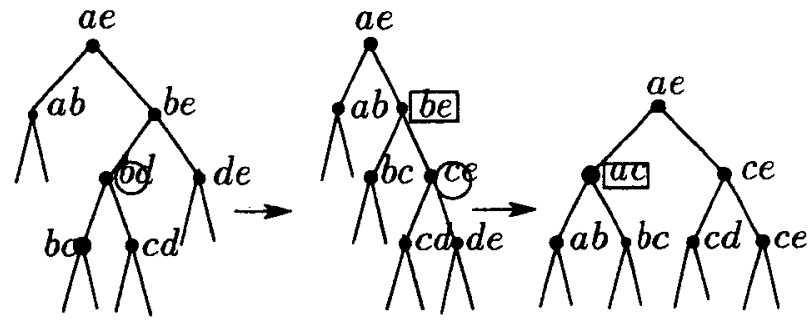

(b)

Figure 12: Illustration of the rebalancing process

Step 3: When all the data points have been introduced, a simple traversal of the resulting contour tree provides the topological contour which is the solution of the contour problem $(P, L)$ if the data are legal. 
Theorem 2 Algorithm Contour provides the topological contour solution of a legal set of data $(P, L)$ of size $n$ in time $O(n \log n)$. The data structure uses $O(n)$ space and may be updated in time $O(\log n)$ when adding or deleting a point from the data set. These results are asymptotically optimal in the worst-case.

Proof : From the above discussion, it is clear that Algorithm Contour provides the topological solution of the contour problem $(P, L)$ if the data are legal. The analysis of the worst case complexity of this algorithm is straightforward. Step 1 is a call to the function Threepoints which, from Lemma 3, takes constant time and the initialization of the data structure is obviously also performed in constant time. In Step 2, the insertion of each new point causes first a path to be followed from the root to a leaf of the contour tree which, since the structure is balanced, causes at most $\mathrm{O}(\log n)$ calls to the function Threepoints. Then, the point is inserted in the structure; the ensuing rebalancing process involves at most $\mathrm{O}(\log n)$ rotations, each of which is performed in constant time. Thus, the overall cost of Step 2 is $O(n \log n)$ time. Step 3 is a simple traversal of the tree, linear in the size of the contour tree which is obviously $\mathrm{O}(n)$. Putting these things together shows that the worst case time complexity of Algorithm Contour is $\mathrm{O}(n \log n)$.

The insertion of a new data point is performed in Step 2 and the above analysis shows that the worst case time complexity of this operation is $O(\log n)$. The deletion of a measured point from the data structure can also be done in $O(\log n)$ time as follows : two paths are followed from the root to the two leaves in the tree where point $x$ appears. These two paths are guided as above by calls to the function Threepoints and they coincide up to a node called the fork. From this node, the point $x$ appear as point $b(V)$ (resp. as point $e(V)$ ) on all the following nodes of the left (resp. right) path. At each occurence, $x$ is replaced by its successor $x^{\prime}$ on the contour. This leaves a trivial leaf labelled $\left(x^{\prime}, x^{\prime}\right)$ which has just to be deleted together with its sibling. Then, the tree is rebalanced. As in the case of an insertion, each path is traversed after at $\operatorname{most} \mathrm{O}(\log n)$ calls to the function Threepoints and the rebalancing process involves at most $\mathrm{O}(\log n)$ rotations which proves the claim.

The optimality of the algorithm comes from the fact that sorting is reducible in $\mathrm{O}(n)$ time to the contour problem. Consider a set of $n$ points $p_{1}, \ldots, p_{n}$ pictured in Figure 13, which consists of $n-1$ collinear points and another, say $q$, not on the same line. Consider the corresponding rays $l_{1}, \ldots, l_{n}, l_{q}$, taken to be parallel half straight lines, normal to the line $p_{1}, \ldots, p_{n} ; l_{1}, \ldots, l_{n}$ lie in the half plane not containing $q$; the direction of $l_{q}$ is opposite to the direction of $l_{1}, \ldots, l_{12}$. The edge list produced by an algorithm solving the contour problem can be used to sort the $p_{i}$ in $O(n)$ additional operations .

\subsection{Testing a posteriori the solution of the contour problem}

If the set of data $(P, L)$ is not legal there exists no solution to the contour problem, not even a topological solution. If the data are legal but not complete, the solution 


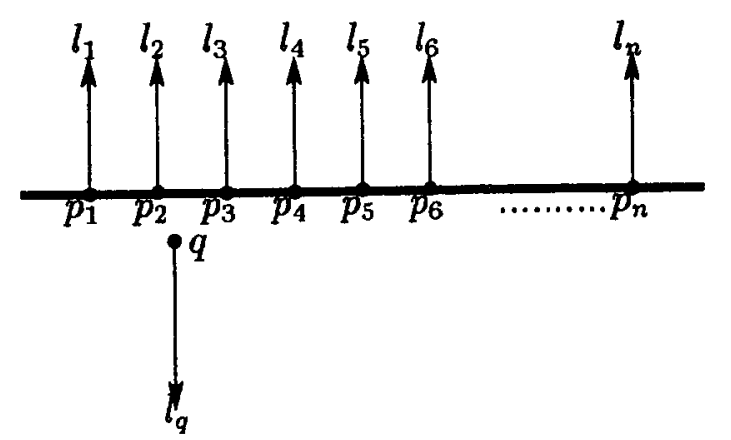

Figure 13: For the lower bound

of the contour problem is only topological and cannot be represented by a simple polygon. In both cases, the ordering obtained by a blind application of the above algorithm corresponds to a polygon which either is not simple or intersects some of the rays. The simplicity of the contour can be tested in $O(n \log n)$ time using a plane sweep algorithm to detect an eventual intersection between the edges, such as the line segment intersection test described by Preparata and Shamos [11].

In order to check if a ray intersects the contour, we use the following result of Chazelle and Guibas [5] : There exists an $O(n)$ space data structure representing a simple polygon $\Pi$ which can be computed in time $O(n \log n)$ and which, given a pair $(p, u)$ of $a$ point $p$ and $a$ direction $u$, can be used to find the first edge of $\Pi$ hit by the ray from $p$ in the direction $u$ in time $O(\log n)$.

We apply this result to all the segments of all the rays of $L$. Let $s$ be such a segment, belonging to some ray $l_{p}$, issued from point $p$; let $a$ be the end-point of $s$ encountered first when the ray is traversed from $p$, and $b$ the other end-point of $s$. We apply the result of Chazelle and Guibas to the pair $(a, a \rightarrow b)$, where $a \rightarrow b$ is the direction of the half-line $\delta$ issued from $a$ and containing $b$, and find in $O(\log n)$ time the first edge, say $e$, of the contour hit by $\delta$. If the intersection between $e$ and $\delta$ belongs to $s$, we have found a ray which intersects the contour; the solution is not valid. Otherwise, we consider another ray segment. Because the number of segments per ray is bounded by a constant, this test takes at most $O(n \log n)$ time. We have shown :

Theorem 3 The validity of the solution to a contour problem can be checked in $O(n \log n)$ time.

Unfortunately, in case of failure of one of the above tests (test for simplicity and test for non intersection with the set of rays), it is not possible in time $O(n \log n)$ to make the distinction between the case of illegal data and the case of incomplete data. 
This point is discussed at length, in a companion paper, for the case of straight line rays [2].

Remark An important practical case of legal but incomplete data arises when the robot has only seen or touched too small a portion of the object (see Figure 14). In that case, the topological solution of the contour problem can be represented by an open polygonal line including all the measured points and closed by an additional curve. In a previous paper [1] we have shown in detail how to slightly modify Algorithm Contour to handle this case.

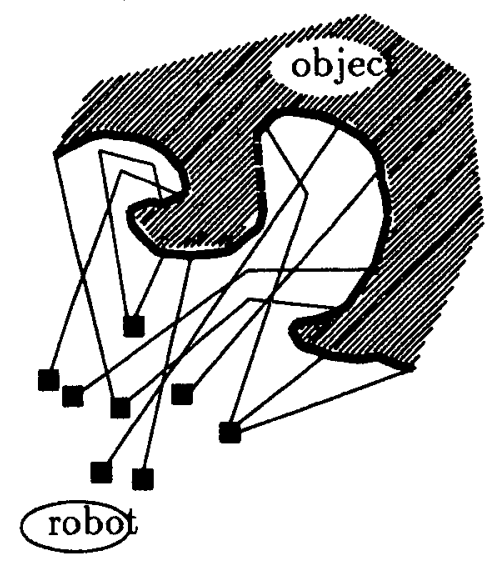

Figure 14: An important case of legal but incomplete data .

\section{Probing simple polygons}

\subsection{Statement of the problem}

Section 2 was concerned with finding a polygonal approximation of an arbitrary object shape from a set of given probes. Though the algorithm of Section 2 was dynamic and allowed insertions and deletions of points, the probes were imposed and the algorithm didn't take care of determining the probes. In this section, we want to discover the exact shape of an object, known to be a simple but not necessarily convex polygon, by means of a minimum (finite) number of on-line probes. "Online" means here that the sequence of probes is determined by the algorithm, and that each individual probe is computed from the knowledge provided by the previous probes.

One probes here along a half line, called the probe path, whose origin is some point $O_{i}$ of the plane. When the probe is issued, the probing device responds with 
the first point $p_{i}$, called the contact point, where the probe path encounters the boundary $C$ of the object and gives also the normal $n_{i}$ to $C$ at $p_{i}$ when it is defined. The sensory device is supposed to be able to detect when $p_{i}$ is a vertex of $C$, in which case the object responds with two normals instead of one, namely the normals to the edges incident to $p_{i}$. An example of such a device may be a finger with a tactile sensor at its tip. In the next subsection (Subsection 3.2), we show that, under some mild conditions which are stated below, we can define a probing strategy which ensures to fully discover the exact shape of the object in at most $3 n-3$ probes where $n$ denotes the number of edges of the object. It is important to realize that $n$ is a priori unknown and will be discovered at the same time as the exact shape of the object. Here are the required conditions :

Condition 1. The oriented supporting lines of the edges of $C$ are all distinct ${ }^{3}$. Notice that two supporting lines may be identical if their orientations are opposite.

Condition 2. A point in the interior of $C$ is given. Without loss of generality, we take this point as the origin.

These two conditions are made to ensure that the probing problem is solvable in a finite number of steps. Indeed, without the first condition, a small detail of the object may still have been missed after any finite number of probes. Another way to circumvent this difficulty, that we do not follow here, would be to assume that the edges of the object have at least a minimal finite length. The second condition allows one to isolate the problem of discovering the shape of the object from the problem of locating it within the workspace. Without this condition, we have no idea where $C$ is located and an unbounded number of probes can be required to find it.

In the following subsections, we show that each successive probe needed in this strategy can be determined in time $O(\log n)$ (Subsection 3.3) and that our strategy is optimal in the sense that $3 n-3$ probes may be actually necessary in some adversary worst case (Subsection 3.4).

The kind of response assumed here requires a powerful probing device; previous work on the subject $[6,12]$ has assumed simpler probes, most notably "finger probes", which return only the first contact point. In Section 3.5, we show that, under a mild condition on the ability of the probing device, our algorithm can work for those simple probes. More precisely, we show that, under that condition, $5 n-2$ probes are sufficient to discover the shape of the object in this case and that, without this condition, $5 n-2$ probes allow to discover a shape which is almost surely the actual shape of the object.

\footnotetext{
${ }^{3} C$ is supposed to be oriented counterclockwise and the edges and their supporting lines accordingly.
} 
A last point about this problem, that is not considered here but will be in a forthcoming paper, is the problem of optimizing the trajectory of the robot. Indeed, we show here that each new probe can be computed in time $O(\log n)$ but that does not presume anything about the time needed by the robot to move to the right position $O_{i}$ and to execute such a probe, except if the robot can jump from one position to another in $O(1)$ time, as can do a robot moving in 3 -d space but constrained to probe in a plane, in which case each probe can be specified and executed in $O(\log n)$ time.

\subsection{Upper bound on the number of probes}

Our probing strategy is based on the use of the total order induced on the set of contact points by the set of probed paths. To make use of the results of the previous section, each new probe is chosen so that the outcoming contact point $p_{i}$ can be associated with a semi-infinite ray $l_{i}$ ending at $p_{i}$ and known not to intersect the interior of the object. The origin $O_{i}$ of the current probe path is chosen to be either a point at infinity or to belong to a previous probe path. Ray $l_{i}$ is the concatenation of a prefix (made of portions of previous probe paths) and of an extra segment, the portion of the current probe path connecting $O_{i}$ to $p_{i}$. In the sequel, we shall consider that a probe outcome, noted $\varpi_{i}=\left(p_{i}, n_{i}, l_{i}\right)$, includes three components : the contact point $p_{i}$, the normal $n_{i}$ to the boundary $C$ of the object at $p_{i}$ and the semi-infinite ray $l_{i}$ ending at $p_{i}$.

Given a probe outcome $\varpi_{i}=\left(p_{i}, n_{i}, l_{i}\right)$, we call the line $D_{i}$, normal to $n_{i}$ and passing through $p_{i}$, the supporting line of $\varpi_{i}$. When necessary, $D_{i}$ will be oriented so as to let $l_{i}$ on its right side in the neighborhood of $p_{i}$. If $p_{i}$ belongs to the edge $e_{j}$ of $C$, we say that $e_{j}$ has been discovered.

Let us consider a set of $s$ probes whose outcomes $\varpi_{1}, \ldots, \varpi_{s}$ are indexed according to the order induced by the corresponding rays $l_{1}, \ldots, l_{s}$. If at least one probe had been performed on each edge of $C$, it would be easy to obtain $C$ by the following simple procedure $\mathrm{A}$, which computes a list $V$ containing the ordered set of vertices of $C$ (indices are taken modulo $s$ ):

\section{Procedure A}

1. $V:=\emptyset$;

2. for $i=1, \ldots, s$ do if $D_{i} \neq D_{i+1}$ then add $D_{i} \cap D_{i+1}$ to $V$;

3. end.

However, if some edges have not been discovered yet, Procedure A yields vertices that do not belong to the object. Roughly speaking, our strategy consists in issuing probes that aim these potential vertices in order to either confirm them as actual vertices of the object or to discover new edges. 
We start with three probes. The first two probes are performed along straight line rays with opposite directions and both passing through the origin. Let $D_{1}$ and $D_{2}$ denote the two supporting lines of the two corresponding probe outcome $\varpi_{1}$ and $\varpi_{2}, I=D_{1} \cap D_{2}$ (possibly at infinity). The third probe is performed along a directed straight line passing through the origin and $I$ (directed in such a way that the origin is reached before $I$ ). The three corresponding contact points $p_{1}, p_{2}, p_{3}$ belong to three distinct edges of $C$.

At a given stage of the algorithm some edges have been discovered. The rays associated with the probes induce an ordering of the contact points and also of the discovered edges (the ordering along $C$ ). The intersection $I$ between the supporting lines $D_{1}$ and $D_{2}$ of two successive contact points is called a corner and is a potential vertex of $C$. The algorithm maintains an ordered list of corners $L$ and, at each step, constructs a new probe path which will either confirm the first corner $I$ of $L$ as being a vertex of $C$, or will probe a new point on a not yet discovered edge. In the first case, we simply report the vertex and delete $I$ from $L$; in the latter, two new corners are discovered and are inserted in $L$.

Let $I=D_{1} \cap D_{2}$ be the current first corner of list $L$. The two lines define four wedges $R$ (with $p_{1}$ and $p_{2}$ on its boundary), $S$ (with $p_{1}$ but not $p_{2}$ on its boundary), $T$ (with neither $p_{1}$ nor $p_{2}$ on its boundary) and $U$ (with $p_{2}$ but not $p_{1}$ on its boundary). Let $\varpi_{1}=\left(p_{1}, l_{1}, n_{1}\right)$ and $\varpi_{2}=\left(p_{2}, l_{2}, n_{2}\right)$ be the two probe outcomes whose supporting lines are $D_{1}$ and $D_{2}$ and let $e_{1}$ and $e_{2}$ be the edges of $C$ containing $p_{1}$ and $p_{2}$ respectively. The two points $p_{1}$ and $p_{2}$ are adjacent in the order induced by the set of rays, at this stage of the algorithm. Therefore, from Lemma 1 , the region $H_{12}$ limited by the ray $l_{1}$, the ray $l_{2}$, the portion $C_{12}$ of the boundary of $C$ between $p_{1}$ and $p_{2}$, and lying to the right when traversing $C_{12}$ from $p_{1}$ to $p_{2}$, is known to contain no contact point of the previous probes and no confirmed vertex. Furthermore, the contact point $p$ of a probe $\left(p \neq p_{1}\right.$ and $\left.p \neq p_{2}\right)$, is to be inserted between $p_{1}$ and $p_{2}$ on the boundary of $C$ if and only if $p$ lies inside $H_{12}$.

Notice that $p$ cannot belong to $l_{1}$ nor to $l_{2}$ since otherwise it would have been already found by a previous probe.

We denote by $h_{12}$ the set of points which belong to the boundary of $H_{12}$ (considered as a closed region) but not to $C_{12} . h_{12}$ is considered to be a connected curve (which may include points at infinity) oriented from $p_{2}$ to $p_{1}$.

Our aim now is to exhibit probe paths that will either confirm $I$ as being a vertex of $C$ or discover a new edge of $C$. Let $D$ be a straight line passing through $I$ and contained in $R \cup T . D$ intersects the segment $p_{1} p_{2}$. We orient $D$ so that $p_{1}$ is on the left side of $D$ and $p_{2}$ on its right side. Let $\gamma=h_{12} \cup C_{12} . \gamma$ is a simple closed curve (possibly containing points at infinity). From the Jordan theorem, $D$ intersects $\gamma$ in an even number of points $O_{1}, \ldots, O_{2 k}{ }^{4}$. In the case that $h_{12}$ contains the point at infinity of $D$, one intersection point is at infinity: we take it to be $O_{1}$. Let $\tau_{i}$

\footnotetext{
${ }^{4}$ We assume that $D$ intersects $\gamma$ properly ; otherwise, we slightly move $D$.
} 
$(i=1, \ldots, 2 k)$ be the vector tangent to $\gamma$ at $O_{i}$, oriented in the same way as $h_{12}, C_{12}$ and thus $\gamma$. To each intersection point $O_{i}(i=1, \ldots, 2 k)$, we associate a sign, + or - , according as the orientation of the frame $\left(D, \tau_{i}\right)$ is positive or not (see Figure 15). From the Jordan theorem, the sequence of signs $\Sigma(\gamma)$ of the intersections between $\gamma$ and $D$, sorted along $D$, is an alternating sequence of + and $-:+-+-+-\ldots+-$.

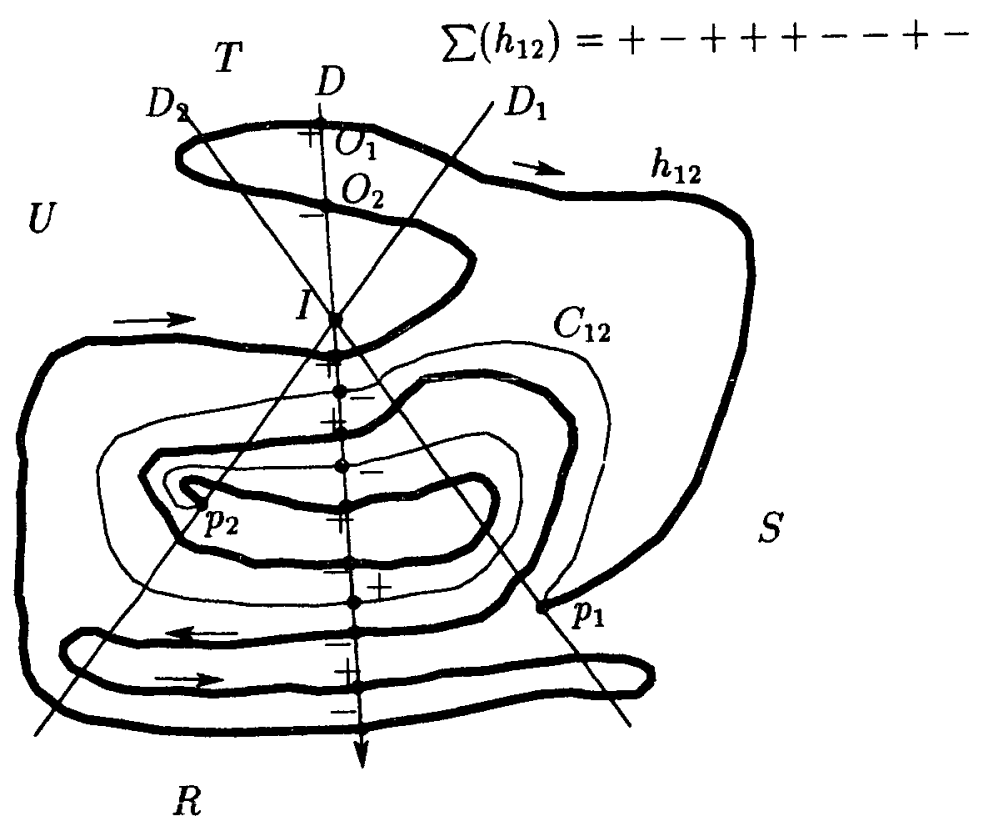

Figure 15: The sequence of signs

$h_{12}$ is a simple piece of curve joining the two points $p_{1}$ and $p_{2}$. Because $p_{1}$ and $p_{2}$ are on different sides of $D$, it follows from Jordan theorem that $h_{12}$ intersects $D$ in an odd number of points. Let $\Sigma\left(h_{12}\right)$ be the corresponding sequence of signs. The number of + signs in $\Sigma\left(h_{12}\right)$ is exactly one more than the number of - signs. Moreover, $\Sigma\left(h_{12}\right)$ necessarily starts with a + sign because $H_{12}$ lies on the right side of $C_{12}$ (oriented from $p_{1}$ to $p_{2}$ ). and thus $D$ intersects $h_{12}$ before it intersects $C_{12}$. Hence, $\Sigma\left(h_{12}\right)$ necessarily terminates by + sign (Case 1 ) or contains two consecutive $+\operatorname{sign}$ (Case 2). Because the sequence of signs of $\gamma$ is an alternating sequence of + and -, there exist in both cases two intersection points, successive along $D$, such that the first one is an intersection point between $D$ and $h_{12}$ and has + sign and the second one is an intersection between $D$ and $C_{12}$ and has - sign. We rename these two points $O$ and $p$ respectively. Notice that, in Case $1, O$ is the last intersection point between $h_{12}$ and $D$; in Case $2, O$ is the first of two successive intersection 
points with signs + ; thus $O$ can be determined from $h_{12}$ and $D$.

Let $\mu$ be the half line supported by $D$, with the same orientation as $D$ and starting at $O$. We associate with $\mu$ a probing ray $l$ which is exactly the portion of $\mu$ between $O$ and $p$ if $O$ is a point at infinity and, otherwise, the concatenation of $\mu$ with the infinite portion of the ray $l_{i}(i=1$ or 2$)$ passing through $O$. From the above discussion, it is clear that $\mu$ intersects the boundary of $C$ for the first time at point $p$ and that $p$ belongs to $H_{12}$. So $p_{1}, p, p_{2}$ are encountered in that order along the boundary of $C$. Let $\varpi=(p, l, n)$ be the corresponding probe outcome.

We distinguish four possible cases, depending whether $p$ belongs to $e_{1}, e_{2}$, both or none. Notice that, due to Condition 1 above, $p$ belongs to $e_{i}$ iff $p$ belongs to $D_{i}$ and $n=n_{i}$.

\section{Case 1: $p \in e_{1}$ and $p \in e_{2}$}

In this case, $p=I . I$ is confirmed as a vertex of $C$. Due to Condition 1 , we are guaranteed that the edges containing $p_{1}$ and $p_{2}$ are adjacent along $C$ and that $I$ is their common vertex.

\section{Case 2: $p \notin e_{1}$ and $p \notin e_{2}$}

The supporting line $D(\varpi)$ of the probe outcome is distinct from $D_{1}$ and $D_{2}$. Moreover, it is distinct from all the supporting lines of the previous probes, which means that we have discovered a new edge. Indeed, let us suppose the contrary. Let $\varpi^{\prime}=\left(p^{\prime}, l^{\prime}, n^{\prime}\right)$ be a probe outcome whose supporting line $D\left(\varpi^{\prime}\right)=D(\varpi)$. Due to Condition 1 above, the contact points $p$ and $p^{\prime}$ belong to the same edge of $C$. Moreover, due to the construction of $\mu, p \in H_{12}$ and so we have $p_{1}<p<p_{2}$. Thus we have also $p_{1}<p^{\prime}<p_{2}$, which contradicts the fact that $p_{1}$ and $p_{2}$ are consecutive in the order induced by the rays.

\section{Case 3: $p \in e_{1}$ and $p \notin e_{2}$}

In this case $p=I$ but is not a vertex of $C$. Thus we have not confirmed $I$ as a vertex of $C$ and we have not discover any new edge. We need another probe. Let $\Pi_{1}$ be the half-plane on the right side of $D_{1}$, when oriented as described in Section 3.1. We distinguish two cases according to wether $p_{2}$ belongs to $\Pi_{1}$ or not. In both cases, we exhibit a new probe path $\mu^{\prime}$ which is guaranteed to discover a new edge of the boundary of $C$ between $p_{1}$ and $p_{2} . \mu^{\prime}$ will be supported by a straight line $D^{\prime}$ passing through $I$ and contained in $S \cup U$.

\section{Subcase 3.1: $p_{2} \in \Pi_{1}$}

The situation is depicted in Figure 16. In this case, $D^{\prime}$ is oriented from $S$ to $U$. Let $\mu^{\prime}$ be the half line supported by $D^{\prime}$ and starting at $I$. The contact point probed by 
$\mu^{\prime}$ is $p^{\prime}$. The corresponding ray $l^{\prime}$ is the concatenation of $I p^{\prime}$ and $l$. Using arguments similar to those of Case 2 , the new probe necessarily discovers a new edge of $C$ (between $p_{1}$ and $p_{2}$ ).

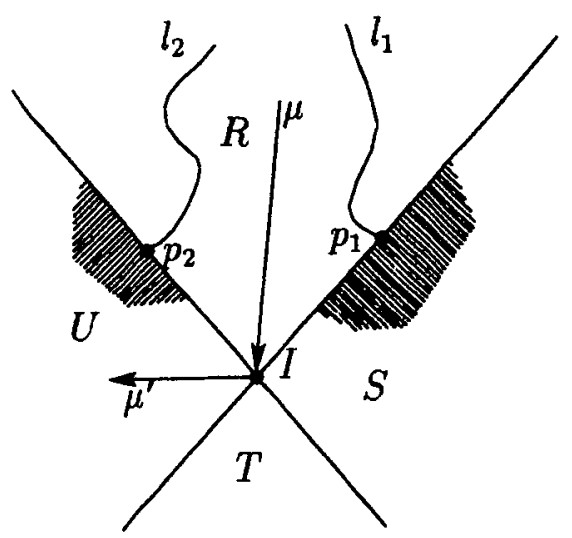

Figure 16: Case 3.1

\section{Subcase 3.2: $p_{2} \notin \Pi_{1}$}

The situation is depicted in Figure 17. We now orient $D^{\prime}$ from $U$ to $S$. As in Case 2 , among the intersections between $D^{\prime}$ and the closed Jordan curve $h_{12} \cup C_{12}$, there exist two successive intersection points along $D^{\prime}$ such that the first point, $O^{\prime}$, is an intersection point with + sign between $D^{\prime}$ and $h_{12}$ and the second one, $p^{\prime}$, is an intersection point with - sign between $D^{\prime}$ and $C_{12}$. We take $O^{\prime}$ as the origin of $\mu^{\prime}$. The contact point probed by $\mu^{\prime}$ is $p^{\prime}$. The corresponding ray $l^{\prime}$ is simply $O^{\prime} p^{\prime}$ if $O^{\prime}$ is at infinity or the concatenation of $O^{\prime} p^{\prime}$ with the infinite part of the ray passing through $O^{\prime}$ otherwise. Using arguments similar to those of Case 2, the new probe necessarily discovers a new edge of $C$ (between $p_{1}$ and $p_{2}$ ).

Case 4: $p \notin e_{1}$ and $p \in e_{2}$

This case is analogous to the previous one. The indices 1 and 2 have simply to be exchanged as well as the wedges $U$ and $S$.

In conclusion, each time a corner is checked, we either confirm the corner as a vertex of $C$ by means of one probe and this corner will never be probed again or we discover a new edge by means of at most two probes. Thus to discover $C$ we need at most one probe per vertex and two probes per edge, except for the first three edges 


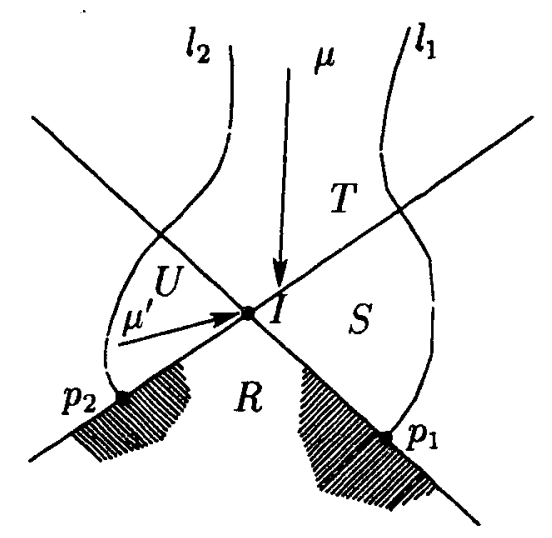

Figure 17: Case 3.2

which are discovered by means of only one probe each. This proves the following theorem :

Theorem $43 n-3$ probes are sufficient to determine the exact shape of a simple polygon with $n$ non colinear edges.

\subsection{Complexity analysis}

The above strategy guaranties that a finite number of probes are performed. However, in order to achieve an effective algorithm, we need to make precise how to construct the probe paths. We show now that we can restrict ourselves to some polygonal rays and that each ray can be determined in $O(\log n)$ time yielding an overall $\mathrm{O}(n \log n)$ time algorithm.

More precisely, at each step, the new probe path $\mu$ and, eventually, the additional probe path $\mu^{\prime}$ are constructed as described in the previous section. We take a straight oriented line $D$ contained in $R \cup T . \mu$ is a half line supported by $D$, with the same orientation as $D$. Its origin $O$ is an intersection point between $D$ and $h_{12}$ with sign + which either is the last intersection between $D$ and $h_{12}$ or immediately precedes another intersection between $D$ and $h_{12}$ with sign + . The additional probe path $\mu^{\prime}$, if needed, is taken to be supported by a straight line $D^{\prime}$ contained in $S \cup U$. The origin $O^{\prime}$ is either the corner $I$ (Subcases 3.1 and 4.1 ) or a point defined in a way similar to the way $O$ has been defined above (we simply have to exchange the roles of $D$ and $D^{\prime}$ ).

Lemma 4 The probes can be constructed in such a way that, at each step of the algorithm, $h_{12}$ is a polygonal convex curve (i.e., the angle between two successive 
segments of $h_{12}$, in the order they are encountered when going from $p_{2}$ to $p_{1}$, is less than 180 degrees).

Proof: The proof is by induction on the number of steps (one step corresponding to one of the cases $1,2,3$ or 4 ).

Clearly, the lemma holds after the initialization. Let us suppose that $k$ steps have been performed and that the above claim holds. Let us consider now step $k+1$.

Assume first that Step $k+1$ corresponds to Case 1 or 2. Once the origin $O$ of $\mu$ has been chosen (as described in the previous section), $\mu$ is a half line with a point $O$ of $h_{12}$ as its origin and lying on the right side of $h_{12}$ (oriented as usual from $p_{2}$ to $p_{1}$ ), in the neighborhood of $O$. The new probe path $\mu$, ending at the contact point $p$, splits the polygonal line $h_{12}$ into two new polygonal lines $h_{1 p}$ and $h_{p 2}$. The angles arising on each of those lines are exactly the angles of $h_{12}$ except at vertex $O$ where the angle arising on $h_{1 p}$ and the angle arising on $h_{p 2}$ sum to the angle arising on $h_{12}$. Thus these two new angles are also convex. The same arguments hold for both probes in Subcases 3.1 and 3.2 and similarly in Subcases 4.1 and 4.2. The first probe path splits the polygonal chains $h_{12}$ into two polygonal chains $h_{1 I}$ and $h_{I 2}$. The second probe $\mu^{\prime}$ will further split either $h_{I 2}$ (Subcases 3.1 and 3.2) or $h_{1 I}$ (Subcases 4.1 and 4.2) into two convex polygonal chains. This completes the proof.

A direct consequence of the fact that $h_{12}$ is a convex curve is that, either $D$ intersects $h_{12}$ only once or the first two intersections between $D$ and $h_{12}$ along $D$ have + signs . Similarly, in Subcases 3.2 and 4.2, either $D^{\prime}$ intersects $h_{12}$ only once or the first two intersections between $D^{\prime}$ and $h_{12}$ along $D^{\prime}$ have + signs. This proves the following lemma:

Lemma 5 We can take, as the origin of $\mu$, the point of intersection between $h_{12}$ and $D$ first encountered when marching along D. In Subcases 3.2 and 4.2, we can take, as the origin of $\mu^{\prime}$, the point of intersection between $h_{12}$ and $D^{\prime}$ encountered first when marching along $D^{\prime}$.

Let $\mathcal{H}$ be the current set of polygonal chains $h_{i, i+1}$ between pairs of points on the boundary of $C$ that are consecutive at this stage.

Lemma $6 \mathcal{H}$ can be stored in a dynamic structure of size $O(n)$ such that:

1. The first intersection between an oriented line $D$ (or $D^{\prime}$ ) and a polygonal chain $h_{i, i+1}$ can be found in $O(\log n)$ time;

2. The structure can be updated in $O(\log n)$ time after each new probe.

Proof : Let us first describe the data structure used to store set $\mathcal{H}$. Each chain $h_{i, i+1}$ is considered as the concatenation of two subchains $h_{i, i+1}^{i}$ and $h_{i, i+1}^{i+1} . h_{i, i+1}^{i}$ (resp., 
$h_{i, i+1}^{i+1}$ ) consists of the portion of $h_{i, i+1}$ belonging exclusively to the ray $l_{i}$ (resp., $l_{i+1}$ ). Let $s_{p_{i}, p_{i+1}}$ be the point of $h_{i, i+1}$ where rays $l_{i}$ and $l_{i+1}$ separate. The two subchains $h_{i, i+1}^{i}$ and $h_{i, i+1}^{i+1}$ are themselves decomposed into an ordered list of subchains (called subsubchains) as follows. The first subsubchain is the polygonal line between $s_{p_{i}, p_{i+1}}$ and the first local extremum with respect to the $x$-axis, encountered when marching along the chain from $s_{p_{i}, p_{i+1}}$ towards the measured point. The last subsubchain is the polygonal line between the last local extremum and the measured point. The other subsubchains join two successive local extremum. Figure 18 illustrates these definitions : $h_{12}$ is decomposed into two subchains $h_{12}^{1}=s_{p_{1}, p_{2}} \rightarrow p_{1}$ and $h_{12}^{2}=s_{p_{1}, p_{2}} \rightarrow$ $p_{2} . h_{12}^{1}$ is decomposed into five subsubchains, $s_{p_{1}, p_{2}} \rightarrow e_{1}^{1}, e_{i}^{1} \rightarrow e_{i+1}^{1}(i=1,2,3)$, $e_{4}^{1} \rightarrow p_{1} . h_{12}^{2}$ is decomposed into five subsubchains, $s_{p_{1}, p_{2}} \rightarrow e_{1}^{2}, e_{i}^{2} \rightarrow e_{i+1}^{2}(i=$ $1,2,3), e_{4}^{2} \rightarrow p_{2}$. Each of these subsubchains is stored in a concatenable queue.

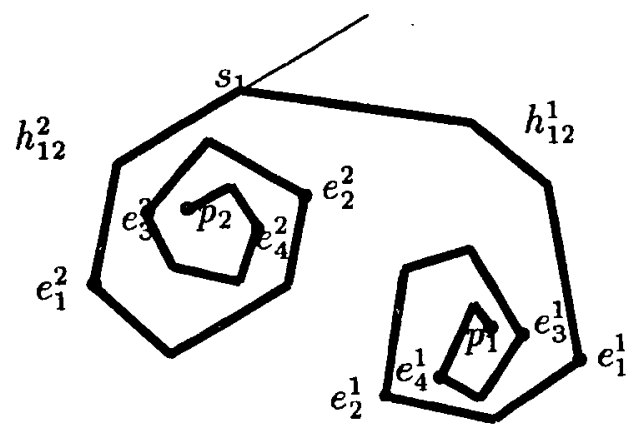

$X-a x i s$

Figure 18: For the notion of subsubchains

Since the total number of segments of the polygonal chains $h_{i, i+1}$ is $O(n)$, the whole data structure can be implemented so as to require $O(n)$ space.

Let us show that this structure allows us to achieve the two goals of the Lemma. Let $D$ be an oriented line. Since $h_{i, i+1}^{i}$ and $h_{i, i+1}^{i+1}$ are convex, it is clear that $D$ intersects each new subsubchain in at most two points and that these intersections can be computed in $O(\log n)$ time by binary search. Furthermore, we claim that the first intersection between $D$ and $h_{i, i+1}$, encountered when marching along $D$, belongs to the first three subsubchains of either $h_{i, i+1}^{i}$ or $h_{i, i+1}^{i+1}$. Indeed, let us consider the convex hull $C H\left(h_{i, i+1}\right)$ of the chain $h_{i, i+1}$ :

1. The point $s_{p_{i}, p_{i+1}}$ belongs to $C H\left(h_{i, i+1}\right)$ as is easily shown by induction.

2. From the convexity of $h_{i, i+1}, C H\left(h_{i, i+1}\right)$ is in fact a part of $h_{i, i+1}$ plus one 


\section{bridging edge.}

3. Because $D$ intersects the segment $p_{i} p_{i+1}$ leaving point $p_{i}$ on its left side and $p_{i+1}$ on its right side ${ }^{5}, D$ intersects $h_{i, i+1}$ for the first time in the portion of this chain which belongs to $C H\left(h_{i, i+1}\right)$.

4. The portion of $h_{i, i+1}$ which belongs to $C H\left(h_{i, i+1}\right)$ can only include edges of the first three subsubchains of $h_{i, i+1}^{i}$ and $h_{i, i+1}^{i+1}$.

This proves the claim. Thus to find the first intersection of $D$ with $h_{i, i+1}$, it is sufficient to search the three first concatenable queues of $h_{i, i+1}^{i}$ and $h_{i, i+1}^{i+1}$. This proves the first part of the lemma.

Let $p$ be a new contact point, between $p_{i}$ and $p_{i+1}$. The data structure has to be updated in order to include the four subchains $h_{p_{i}, p}^{p_{i}}, h_{p_{i}, p}^{p}$ (corresponding to the pair $\left.\left(p_{i}, p\right)\right)$ and $h_{p, p_{i+1}}^{p}, h_{p, p_{i+1}}^{p_{i+1}}$ (corresponding to the pair $\left.\left(p, p_{i+1}\right)\right)$ and to remove the subchains $h_{i, i+1}^{i}$ and $h_{i, i+1}^{i+1}$. Without loss of generality, assume for instance that the point $O$ where the ray measuring $p$ separates from $l_{i}$ or $l_{i+1}$, belongs to $h_{i, i+1}^{i}$. To update the data structure, we need to perform the following steps:

1. The polygonal chain $h_{i, i+1}^{i}$ is cut into two parts at point $O$. This entails splitting the concatenable queue associated with the subsubchain containing $O$.

2. The new chain $h_{p_{i}, p}^{p_{i}}$ is the part of $h_{i, i+1}^{i}$, denoted $h_{i, i+1}^{i(2)}$, which joins $O$ to $p_{i}$.

3. The chain $h_{p_{i}, p}^{p}$ consists of only one subsubchain, namely the segment $O p . s_{p_{i}, p}$ is identical to $O$.

- 4. The chain $h_{p, p_{i+1}}^{p}$ is formed by concatenating the first part $h_{i, i+1}^{i(1)}$ of $h_{i, i+1}^{i}$ (joining $s_{p_{i}, p_{i+1}}$ to $O$ ) and segment $O p . s_{p_{,} p_{i+1}}$ is identical to $s_{p_{i}, p_{i+1}}$. In this operation, we have to check whether or not $O$ is a local extremum of $h_{p, p_{i+1}}^{p}$. If $O$ is not a local extremum, $O p$ is simply appended to the last subsubchain of $h_{i, i+1}^{i(1)}$; if $O$ is a local extremum, $O p$ itself is a subsubchain, namely the last subsubchain in the list of subsubchains of $h_{i, i+1}^{i(1)}$.

5. The chain $h_{p, p_{i+1}}^{p_{i+1}}$ is simply the previous chain $h_{p_{i}, p_{i+1}}^{p_{i+1}}$.

Checking for a local extremum can be done in constant time while splitting or concatenating concatenable queues can be done in $O(\log n)$ time. Thus, updating the data structure, after the probing of a new point $p$, can be done in $O(\log n)$ time. This proves the second part of the lemma.

We can state now the main result of this section :

\footnotetext{
${ }^{5}$ In the case of the additional probe $\mu^{\prime}$, needed in Case 3.2 (resp., Case 4.2), the same argument holds, provided that we rename $p_{i}$ (resp., $p_{i+1}$ ) the corner $I$, intersection of $D_{i}$ and $D_{i+1}$.
} 
Theorem 5 Each of the at most $3 n-3$ probes can be determined in $O(\log n)$ time yielding an algorithm with overall complexity $O(n \log n)$ time and $O(n)$ storage for discovering the exact shape of a simple polygon with $n$ non colinear edges.

Proof : In each of the cases 1, 2 or 3 of Section 3.2, the probe path $\mu$ is defined as soon as its origin $O$ is found. From Lemma 6, the origin $O$ of $\mu$ and thus $\mu$ itself can be computed in $O(\log n)$ time. In Subcase 3.1 (resp., 4.1), the second probe $\mu^{\prime}$ can be taken to be the bissector of $U($ resp., $S)$ with its origin at $I$ and thus can be computed in constant time. In Subcases 3.2 and 4.2 , it follows from Lemma 3 that the origin $O^{\prime}$ of $\mu^{\prime}$ and thus $\mu^{\prime}$ itself can be computed in $O(\log n)$ time.

When a probe with outcome $\varpi=(p, l, n)$ discovers a new edge between two edges $e_{1}$ and $e_{2}$, the two pairs $\left(p_{1}, p\right)$ and $\left(p, p_{2}\right)$ are new pairs of consecutive points in the order induced by the rays. Thus updating the list of corners takes constant time.

For each pair of consecutive contact points (in the order induced by the rays, the corresponding polygonal line $h_{12}$ is stored as a concatenable queue. Updating the data structure, when the new polygonal lines $h_{1 p}$ and $h_{p 2}$ are constructed, can be done in $O(\log n)$ time. With Theorem 1 , we conclude that the overall time complexity of the algorithm is $\mathrm{O}(n \log n)$ time.

At each step of the algorithm, the number of ray segments is equal to the number of contact points already probed which is known to be less than $3 n-3$. Thus the total number of edges of all the polygonal lines $h_{i, i+1}$ is at most $6 n-6$ and the storage complexity of the algorithm is $\mathrm{O}(n)$.

It is to be noticed that Theorem 5 holds though we cannot bound by a constant the number of segments of a ray, which may be $O(n)$. This is obtained by expressing our probes in an implicit way, as the concatenation of a prefix plus an extra segment. Specifying all probes individually would require $\Omega\left(n^{2}\right)$ space and time.

\subsection{Lower bound on the number of probes}

Polygon $C$ is not completely explored as long as we do not have a contact point on each edge and a contact point at each vertex for, otherwise, we might have missed an edge of $C$ (of potentially arbitrary small size). Thus a trivial lower bound on the number of probes needed to discover a $n$-sided polygon is $2 n$. However, we show, in this section, that whatever the probing strategy may be, $3 n-3$ probes may be necessary in the worst-case, which proves that the results of the two previous sections are optimal.

Let us consider a probing strategy $S$ that tries to discover the exact shape of $C$ by means of a sequence of probes. Suppose that $i-1$ probes have already been performed. At this stage, some edges of $C$ have been discovered. The rays associated with the probes induce an ordering of the discovered edges (the same as the one on 
$C)$. As in Section 3.2, the intersection $I$ between the supporting lines $D_{1}$ and $D_{2}$ of two successive contact points is called a corner and is a potential vertex of $C$. Either this corner is a vertex of $C$ or some new edge has to be discovered between edge $e_{1}$ and edge $e_{2}$. When this corner is not a vertex of $C$ and belongs to only one of the edges $e_{1}$ or $e_{2}$, we can always choose the lengths of $e_{1}$ and $e_{2}$ so that two probes are necessary to discover this new edge. Our objective is to construct a polygon $C$ where this adverse situation is encountered $n-3$ times.

The construction is done by induction on the number of corners which are actual vertices of $C$. Let $\widehat{C}$ be the current estimate of $C$ provided by an application of Algorithm A (i.e., $\widehat{C}$ passes through all the contact points, vertices and corners found so far). At each step, $C$ is chosen so that $\widehat{C}$ satisfies the following induction hypophesis :

Induction hypophesis : All the corners of $\widehat{C}$ are actual vertices of $C$ except one, which is a convex corner (a corner between two successive points $p_{i}$ and $p_{i+1}$ is convex if the angle $\left(n_{i}, n_{i+1}\right)$ between the oriented normals is in $] 0, \pi[\operatorname{modulo} 2 \pi)$.

In the best case, three initial probes $p_{1}, p_{2}, p_{3}$ belong to three distinct edges. We can always choose $C$ so that the three corners are convex, two of them being actual vertices of $C$ - a situation which fulfills the hypothesis.

Let us suppose that, at a given stage, $\widehat{C}$ has $k+1$ vertices and satisfies the induction hypothesis. Let $I$ be the convex corner of $\widehat{C}$ which is not a vertex of $C$. $I$ is the intersection of the supporting lines of $D_{1}$ and $D_{2}$ of the successive contact points $p_{1}$ and $p_{2}$. We can always choose $C$ so that the two following conditions are satisfied :

1. at least two probes are necessary to discover a new edge $e$ (with supporting line $D_{e}$ ) between $p_{1}$ and $p_{2}$;

2. one of the two new corners $\left(D_{1}, D_{e}\right)$ or $\left(D_{e}, D_{2}\right)$ is actually a vertex of $C$ while the other is a convex corner.

Let $\mu$ be the probe aiming at discovering $e$. We first notice that the origin of $\mu$ cannot belong to $\widehat{C}$, otherwise we would choose $C$ to contain also this origin. Moreover, the inductive hypothesis implies that, from $p_{2}$ to $p_{1}$, the boundaries $C_{21}$ and $\widehat{C_{21}}$ of, respectively, $C$ and $\widehat{C}$ coincide. Therefore, if $\mu$ does not intersect the triangle $p_{1} I p_{2}$, $\mu$ either hits the object on $C_{21}$ or we choose $C$ so that $\mu$ misses the object. In both cases, no new edge is discovered. Otherwise, let $p$ be the first intersection between $\mu$ and $p_{1} I p_{2}$. If $p$ is different from $I$ and belongs to $D_{i}(\mathrm{i}=1$ or 2$)$, we take $e_{i}$ to be long enough to contain $p$. Thus no new edge has been discovered. If $p=I$, at least one of the supporting lines $D_{i}(\mathrm{i}=1$ or 2$)$ is oriented so that, in a neighborhood of $I, \mu$ is contained in the half right plane limited by $D_{i}$. We take the corresponding edge $e_{i}$ to be long enough to contain $I$. Again, no new edge has been discovered. 
Thus, in all cases, at least two probes are necessary to discover a new edge between $p_{1}$ and $p_{2}$.

Let us now show that we can choose the edge $e$, which is to be discovered next, in such a way that the above Condition 2 is satisfied. This implies that the inductive hypothesis is restored at the next stage. Remember that, in the adverse situation we consider, either $e_{1}$ or $e_{2}$ contains the corner $I$. Assume that $e_{1}$ contains $I$ while $e_{2}$ does not (the other case is quite similar). Let us choose a point $K$ on the half line supported by $D_{1}$, originating at $I$ and which does not contain $p_{1}$ and let us choose a point $L$ on the segment $p_{2} I$ supported by $D_{2}$. Let us consider the closed curve $\widehat{C^{\prime}}$ formed by the segment $p_{1} K$, the segment $K L$, the segment $L p_{2}$ and the portion $C_{21}$ of the boundary of $C$. Let $\mu^{\prime}$ be a probe that discovers a new edge $e$ between $p_{1}$ and $p_{2}$. The origin of $\mu^{\prime}$ cannot belong to $\widehat{C^{\prime}}$ and moreover, $\mu^{\prime}$ must necessarily intersect $\widehat{C^{\prime}}$; otherwise $C$ is chosen so that $\mu^{\prime}$ misses the object. Moreover, $\mu^{\prime}$ must hit $\widehat{C^{\prime}}$ for the first time at a point $p$ of $K L$; otherwise, either $\mu^{\prime}$ intersects $C_{21}$, in which case no new edge is discovered or $\mu^{\prime}$ intersects $I K$ (resp., $L p_{2}$ ), in which case we choose the length of edge $e_{1}$ (resp., $e_{2}$ ) so that $\mu^{\prime}$ intersects $e_{1}$ (resp., $e_{2}$ ) and thus does not discover any new edge. Point $p$ will be the contact point of the probe $\mu^{\prime}$. The edge $e$ containing $p$ is chosen to be supported by line $(K L)$ and to have $L$ as one of its end points. This implies that $L$ is an actual vertex of $C$. After this probe, all the vertices of the current estimate $\widehat{C}$ are actual vertices of $C$, except for the point $K$, which is a convex corner. This achieves the inductive proof.

Let us evaluate the number of probes necessary to discover $C$. Three initial probes are needed to discover the first three edges of $C$. Then, each new edge requires two additional probes to be discovered. Lastly, $n$ more probes are needed to probe the vertices of $C$. Thus we have:

Theorem 6 Every probe algorithm which determines the shape of a polygon with $n$ edges makes at least $3 n-3$ probes in the worst-case.

\subsection{About the probe model}

It is to be noted that the exact number, $3 n-3$, of probes which are sufficient and necessary, in the worst case, to completely specify the shape of a non convex polygonal object is strongly related to the kind of probe outcomes which is assumed. The probing algorithm developped by Cole and Yap for convex objects assumes a simple finger probe model whose outcome consists only of the coordinates of a point on the boundary of the object but contains no information on the direction of the normal at that point. The main originality of the method presented in this paper is the use of the total order induced on the set of points by the set of rays and one of the interesting questions which may be further raised is : how this order can be used if no information about the direction of the normal at a contact point is available? 


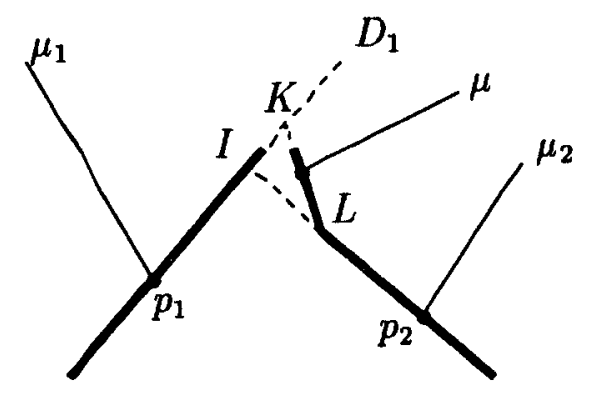

Figure 19: The lower bound

Without additional hypothesis, the problem of probing non convex objects with a finite number of finger probes has no solution. Indeed, if some points have been found on the same straight line, nothing can tell if they actually belong to the same edge of the object or not, even if it is assumed, as above (Condition 1), that the boundary of the object does not include colinear edges. Thus, after any number of probes, small edges of an object may have been missed.

Nevertheless, we show in this subsection that our method can still be applied to the problem of probing without information on the normal directions provided that, in addition to the two conditions stated in Subsection 3.1, the following third condition is fulfilled :

Condition 3. Whenever a probe outcomes includes a point $p$ which is colinear with two previously probed points, the probing device can tell if those three colinear points belong to the same edge of the object or not.

More precisely, we prove the following theorem :

Theorem 7 Provided that Conditions 1, 2 of Subsection 3.1 and Condition 3 above are fulfilled, the exact shape of a non convex polygon can be found with at most $5 n-2$ simple probes. Each probe can be determined in $O(\log n)$ time yielding an algorithm with overall complexity $O(n \log n)$ time and $O(n)$ storage.

Proof: Roughly speaking, we apply to this new probing problem the same strategy as above : at any stage, the algorithm maintains a list of the so far probed points, sorted according to the order induced by the rays, and issues a new ray aiming to probe something at a given place in this order.

An edge is said to be confirmed when at least three points of the edge have been probed and it is said to be discovered when only one or two points of the edge have been probed. It is to be noticed that our probe model cannot detect that a contact point is a vertex. However, if the intersection point of the supporting lines of two confirmed edges has been probed and, thanks to Condition 3, appears to belong to 
both edges, it is necessarily a vertex of the object and will be said to be confirmed as a vertex.

The algorithm begins with three probes which ensure that the three outcoming points belong to at least two distinct edges of the boundary of the object : for instance, the first two probes can be straight lines aiming at the origin $O$ from two opposite directions. Then, the algorithm consider the first pair $\left(p_{1}, p_{2}\right)$ of consecutive contact points which do not belong to the same confirmed edge and distinguish three cases :

Case 1. $p_{1}$ and $p_{2}$ belong to two confirmed edges $e_{1}$ and $e_{2}$ of the boundary of the object. In that case, the supporting lines $D_{1}$ (resp. $D_{2}$ ) of $e_{1}$ (resp. $e_{2}$ ) are known but the corner $D_{1} \cap D_{2}$ is not yet a confirmed vertex and the situation is the same as in the previous problem : a new probe aiming at $D_{1} \cap D_{2}$ is issued and will either confirm this point as a vertex of the object or a new edge lying between $p_{1}$ and $p_{2}$ is discovered by means of at most two probes.

Case 2. One of the points, say $p_{1}$ for instance (the other case is quite symetrical), belongs to a confirmed edge $e_{1}$ while the other, $p_{2}$ does not. Let $D_{1}$ be the oriented line supporting $e_{1}$ and let $\Pi_{1}^{+}$(resp. $\Pi_{1}^{-}$) be the half plane to the left (resp. to the right) of $D_{1}$. If $p_{2}$ lies in $\Pi_{1}^{+}$(resp. in $\Pi_{1}^{-}$), the algorithm issues - in a way similar to that of Section 3.2 - a new probe whose probe path $\mu$ is included in $H_{12} \cap \Pi_{1}^{+}$(resp. in $H_{12} \cap \Pi_{1}^{-}$) : $\mu$ is supported by a line $D$ parallel to $D_{1}$, intersecting the segment $p_{1} p_{2}$ and oriented so that $p_{1}$ lies on its left side and $p_{2}$ on its right side. This new probe yields a contact point $p$ which is between $p_{1}$ and $p_{2}$ on the boundary of the object and does not belong to $e_{1}$. If the three points $p_{1}, p$ and $p_{2}$ or the three points $p, p_{2}$ and the sucessor of $p_{2}$ are found to be colinear and on the same edge, that edge is confirmed, otherwise $p$ belongs to a new edge, not yet discovered.

Case 3. Both points $p_{1}$ and $p_{2}$ do not yet belong to a confirmed edge. In this case, a new probe is issued within $H_{12}$ and the outcoming contact point will either confirm an edge (which may be detected by one of the following triple of points (predecessor of $\left.p_{1}, p_{1}, p\right)$ or $\left(p_{1}, p, p_{2}\right)$ or $\left(p, p_{2}\right.$, successor of $\left.\left.p_{2}\right)\right)$, or discovers a new edge.

In all cases, a new probe will never outcomes a point on a confirmed edge except when a vertex is probed or if a new edge is to be surely discovered by the next probe. As the first three probes discover at least two edges, this ensures that the exact shape of the object is fully discovered in at most $5 n-2$ edges.

As in Section 3.3, each probe is determined by intersecting an oriented line $D$ with a convex curve $h_{12}$. Lemmae 4,5 , and 6 of Section 3.3 still hold, thus proving the complexity result. 
Condition 3 needs some explanation. First of all, let us point out that, because $C$ has no colinear edge, the situation where three points $p_{1}, p_{2}, p_{3}$, not belonging to the same edge of $C$, are colinear is unlikely and not stable, i.e. disappears if we slightly move one of the points along $C$. More precisely, we have the following lemma :

Lemma 7 Let $\tilde{C}$ be the submanifold of the 3-dimensional manifold $C \times C \times C$ consisting of all triples $\left(p_{1}, p_{2}, p_{3}\right)$ of points belonging to $C$ but not to the same edge of $C$. Let $f$ be the mapping

$$
\left(p_{1}, p_{2}, p_{3}\right) \in \tilde{C} \stackrel{f}{\longrightarrow}\left|\begin{array}{cc}
x_{1}-x_{2} & x_{3}-x_{2} \\
y_{1}-y_{2} & y_{3}-y_{2}
\end{array}\right| \in \mathcal{R}
$$

$f^{-1}(0)$ is a 2-dimensional linear submanifold of $\tilde{C}$. In particular, $f^{-1}(0)$ has measure 0 .

Proof : Notice that $f\left(p_{1}, p_{2}, p_{3}\right)=0$ if and only if $p_{1}, p_{2}$ and $p_{3}$ are on a same line. It is plain to show that 0 is a regular value of $f$, i.e. we cannot have simultaneously $f=\delta f=0$. Due to the Preimage theorem [8, page 21], $f^{-1}(0)$ is a submanifold of $\tilde{C}$, with $\operatorname{dim} f^{-1}(0)=\operatorname{dim} \tilde{C}-\operatorname{dim} \mathcal{R}$. As $\tilde{C}$ is a manifold of dimension 3 , we achieve the proof.

Thus, without assuming Condition 3 , we can discover, by means of at most $5 n-2$ probes, a shape that, in almost all cases, will be identical to $C$.

In order to have a purely deterministic algorithm, we need to effectively achieve Condition 3. For any three colinear points, $p_{1}, p_{2}, p_{3}$, this can be done by means of an additional probe along the half line $p_{1} \rightarrow p_{2}, p_{3}$, if we assume further that a probe which goes along an edge and not its interior has no contact point on the edge. In such a case, $6 n-2$ probes will be sufficient.

\section{Conclusion}

This paper has shown that the information provided by the rays is crucial (though generally neglected) when solving 2 -dimensional reconstruction problems. The main property of the rays is that they induce a total order on the measured points when the points belong to a simply connected object. This order has been shown to be computable in optimal time $O(n \log n)$. The algorithm is fully dynamic and allows the insertion or to deletion of a point in $O(\log n)$ time.

From this order a polygonal approximation of the object can be deduced in a straightforward manner. However, if not enough data are available or if the points belong to several connected objects (the data are said to be illegal in this case), this polygonal approximation may not be a simple polygon or may intersect the rays. This can be checked in $O(n \log n)$ time. 
Though we have constrained each ray to comprise a bounded number of line segments, the method still works if the rays each consists of a finite number of simple curves. The complexity results (except for the validity test) hold, provided that a line segment and a curved segment and two curved segments can be intersected in constant time.

The order induced by the rays has also been used to find a strategy for discovering the exact shape of a simple (but not necessarily convex) polygon by means of a minimal number of probes. When each probe outcome consists of a contact point, a ray measuring that point and the normal to the object at the point, we have shown that $3 n-3$ probes are necessary and sufficient if the object has $n$ non colinear edges. Each probe can be determined in $O(\log n)$ time yielding an $O(n \log n)$-time $O(n)$-space algorithm. When each probe outcome consists of a contact point and a ray measuring that point but not the normal, the same strategy can still be applied. Under a mild condition, $5 n-2$ probes are sufficient to discover the shape of the object in this case and, without this condition, $5 n-2$ probes allow to discover a shape which is almost surely the actual shape of the object.

\section{Acknowlegments}

Laurent Baratchart, Richard Cole, Franco Preparata and an anonymous referee are gratefully acknowledged for helpful comments on this paper. Jean-Pierre Merlet is also acknowledged for supplying to us his interactive drawing preparation system JPdraw .

\section{References}

[1] Alevizos P.D., Boissonnat J.D., Yvinec M., An optimal $O(\log n)$ algorithm for contour reconstruction from rays, Proc. 3rd ACM Symp. on Computational Geometry, Waterloo (June 1986).

[2] Alevizos P.D., Boissonnat J.D., Preparata F.P., On the Boundary of a Union of Rays, INRIA Technical Report No. 896 (1988).

[3] Bernstein H.J., Determining the Shape of a Convex n-sided Polygon by using $2 \mathrm{n}+\mathrm{k}$ Tactile Probes, Information Processing Letters 22 (1986) 255-260.

[4] Boissonnat J.D., Geometric structures for three dimensional shape representation, ACM Trans. on Graphics, Vol.3, No 4 (1984), pp. 266-286.

[5] Chazelle B., Guibas L.J., Visibility and Intersection Problems in Plane Geometry, First ACM Symposium on Computational Geometry, Baltimore, June 1985. 
[6] Cole R., Yap C., Shape from probing, Journal of Algorithms 8, 19-38 (1987).

[7] Dobkin D., Edelsbrunner H., Yap C.K., Probing convex polytopes, Proc. ACM Symp. on Theory of Computing (1986), pp. 424-432.

[8] Guillemin V., Pollack A., Differential Topology, Prentice-Hall, 1974.

[9] O'Rourke J., Uniqueness of Orthogonal Connect-the-dots, Dept Comp. Sci., Johns Hopkins University (1986).

[10] O'Rourke J., H. Booth, R. Washington, Connect-the-Dots : A New Heuristic, Comp. Graphics and Image Proc. 39, 1987, pp. 258-266.

[11] Preparata F.P., Shamos M.I., Computational Geometry : an Introduction, Springer Verlag (1985).

[12] Skiena S. S., Geometric Probing, Ph. D. Thesis, Department of Computer Science, University of Illinois at Urbana Champaign, Tech. Report No. UIUCDCSR-88-1425, April 1988. 
